\title{
Psychosocial Effects of Vitiligo: A Systematic Literature Review
}

\author{
Khaled Ezzedine ${ }^{1} \cdot$ Viktoria Eleftheriadou $^{2} \cdot$ Heather Jones $^{3} \cdot$ Kristen Bibeau $^{3} \cdot$ Fiona I. Kuo $^{3} \cdot$ Daniel Sturm $^{3}$. \\ Amit G. Pandya ${ }^{4,5}$
}

Accepted: 27 July 2021 / Published online: 23 September 2021

(c) The Author(s) 2021

\begin{abstract}
Background Patients with vitiligo experience reduced quality of life.

Objective To comprehensively describe the available evidence for psychosocial burden in vitiligo.

Methods A systematic review of observational studies and clinical trials identified using PubMed, EMBASE, Scopus, and the Cochrane databases was performed through 1 March, 2021, to assess psychosocial comorbidities in vitiligo. Two independent reviewers performed an assessment of articles and extracted data for qualitative synthesis.

Results Included studies $(N=168)$ were published between 1979 and 1 March, 2021; 72.6\% were published since 2010. Disorders including or related to depression (41 studies, 0.1-62.3\%) and anxiety (20 studies, 1.9-67.9\%) were the most commonly reported. The most prevalent psychosocial comorbidities were feelings of stigmatization (eight studies, 17.3-100\%), adjustment disorders (12 studies, 4-93.9\%), sleep disturbance (seven studies, 4.6-89.0\%), relationship difficulties including sexual dysfunction (ten studies, 2.0-81.8\%), and avoidance or restriction behavior (12.5-76\%). The prevalence of most psychosocial comorbidities was significantly higher vs healthy individuals. Factors associated with a significantly higher burden included female sex, visible or genital lesions, age $<30$ years (particularly adolescents), and greater body surface area involvement, among others. The most commonly reported patient coping strategy was lesion concealment.

Limitations Available studies were heterogeneous and often had limited details; additionally, publication bias is possible.

Conclusions The results of this systematic review show that vitiligo greatly affects psychosocial well-being. The extent of psychosocial comorbidities supports the use of multidisciplinary treatment strategies and education to address the vitiligoassociated burden of disease.

Protocol Registration PROSPERO (CRD42020162223).

Graphic Abstract

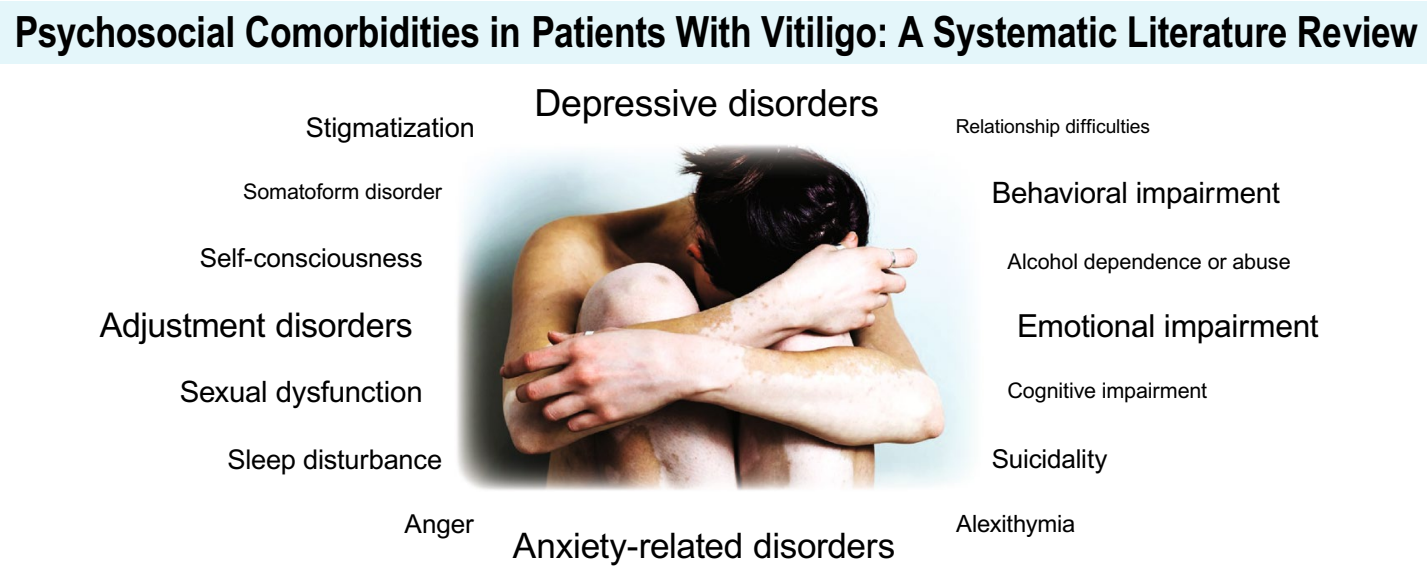

Digital Features for this article can be found at https://doi.org/ 10.6084/m9.figshare.15058539.

Extended author information available on the last page of the article 


\section{Key Points}

Vitiligo has been associated with depression and anxiety; however, other psychosocial comorbidities have not been comprehensively investigated.

A wide variety of psychosocial comorbidities are prevalent in patients with vitiligo; multidisciplinary treatment strategies and education about vitiligo are vital to addressing the burden of this disease.

\section{Introduction}

Vitiligo is a chronic inflammatory autoimmune disease that results in skin depigmentation due to the loss of melanocytes [1-3]. Lesions can appear at any age, but onset usually occurs at $\leq 30$ years of age $[2,4,5]$. Global prevalence is approximately $0.5-2.0 \%$ and varies geographically [6]. Similar prevalence rates have been reported for adult populations as well as children and adolescents [6].

Vitiligo is commonly misinterpreted as a cosmetic disease [2]. Patients with vitiligo experience a higher level of burden [7, 8] compared with healthy controls [7], as reflected by quality-of-life (QoL) indicators. Quality-of-life impairment may be comparable to dermatologic (e.g., atopic dermatitis) $[7,8]$ and non-dermatologic diseases (e.g., cancer) [9]. Importantly, the QoL burden of vitiligo may be largely affected by the presence of psychosocial comorbidities [10-12]. In recent years, a large focus has been placed on depression and/or anxiety in vitiligo [10-12]. The purpose of this systematic literature review was to comprehensively describe the evidence for psychosocial burden in patients with vitiligo, including the prevalence and types of psychosocial comorbidities, factors associated with psychosocial burden, patient coping strategies, perceptions toward vitiligo, and caregiver burden.

\section{Methods}

\subsection{Literature Search}

The search strategy was established and agreed upon by the authors during protocol development (Appendix 1 of the Electronic Supplementary Material [ESM]). PubMed, EMBASE, Scopus, and the Cochrane database were searched for articles from their earliest available entries through 1 March, 2021. The search string, which was limited to articles published in English, included the keywords vitiligo, quality of life, burden, psychosocial, and anxiety, as well as variants of depression, stigma, psychology, and psychiatry. Duplicate results from the separate databases were subsequently discarded.

Peer-reviewed primary publications, including clinical trials and observational studies (cross-sectional, case-control, prospective, and retrospective analyses), were included. Two independent reviewers ( $\mathrm{WvdS}$ and $\mathrm{KW}$ ) performed the title and abstract review; reviews and articles with irrelevant content were excluded. The same reviewers performed the full-text review and data extraction; reviewers independently assessed the risk of bias and resolved any disagreement through discussion. Studies excluded at the full-text review included data sets with fewer than five participants (e.g., patients with vitiligo or their caregivers), editorials, commentaries, articles with irrelevant content (including those that focused only on general QoL and/or that did not report instrument subscales that could be related to psychosocial comorbidity), and articles not available in English. Articles that included the same patient populations but reported different outcomes were retained.

Because data were collected from published articles, no institutional review board approval was required for the study. The study protocol was registered with PROSPERO (CRD42020162223).

\subsection{Data Extraction and Analysis}

Extracted data included study design, geographic region, sample size, detailed patient demographics, prevalence and types of psychosocial comorbidities, extent of psychosocial burden (vs healthy controls and/or patients with other skin diseases), factors associated with psychosocial burden, endpoints (scales) used to assess psychosocial burden, patient coping strategies, perceptions toward vitiligo (by patients and non-patients), and caregiver burden. A qualitative synthesis of evidence was performed to summarize the findings from included primary publications.

\section{Results}

\subsection{Literature Search}

Initial database searches yielded 2288 articles, of which 1111 were duplicate records that were excluded from screening; one additional article was identified through other sources. Screening resulted in the exclusion of 919 articles during the title and abstract review; an additional 91 articles were excluded on the full-text review. A total of 168 articles were retained for data extraction and inclusion in the qualitative synthesis (Fig. 1). 


\subsection{Study Characteristics}

Included studies were published between 1979 and 2021, with $72.6 \%$ published between 2010 and 2021 [4, 5, 9, 13-177]. Most included studies were observational (96.4\%), with only six clinical trials (3.6\%) containing data specific to psychosocial comorbidity in vitiligo; child, adolescent, and adult populations were represented in the included studies (Table 1). Studies representing populations from most geographic regions were included (Fig. 2); regions with the most studies included the Middle East (29.8\%), Europe (28.6\%), and Southern Asia (15.5\%). All studies included in the systematic review were qualitatively assessed to minimize the risk of bias; included studies were deemed to be of acceptable quality.

\subsection{Instruments Measuring QoL}

Among QoL instruments assessing general health, total and/or component scores were most frequently reported for the adult and child versions of the Dermatology Life Quality Index (DLQI, 53 studies) [4, 13, 14, 18, 19, 22, 25, 33, $35-38,45,64,65,67-70,74,77,81-83,87,89,90,92$, 93, 96, 99, 100, 102, 104, 112, 115, 119, 120, 122, 123, $140,143,144,146,148,151,156,161,162,164-166,168]$,
Children's DLQI (seven studies) [24, 67, 73, 101, 103, 110, 152], 36-Item Short Form Health Survey (SF-36, eight studies) $[9,82,83,105-107,161,162]$, General Health Questionnaire (eight studies) [14, 30, 65, 93, 95, 113, 114, 124], and Skindex-29 (eight studies) [29, 71, 98, 106, 107, 162, 174, 175].

The DLQI is widely used across dermatologic diseases. Fifty studies that reported DLQI mean scores for patients with vitiligo were further examined. Mean scores did not differ vastly by region, but there were trends for higher mean scores (i.e., increased burden of disease) in the Middle East (4.7-14.7) [14, 45, 64, 74, 82, 83, 87, 104, 112, 140, 144, 164, 166], Southern Asia (4.1-12.4) [18, 22, 38, 100, 120, 143, 146, 168], and Eastern Asia (4.0-8.4) [35, 68-70, 119, $156,161,162]$ compared with Europe (1.8-8.7) [4, 77, 89, 90, 92, 93, 96, 99, 102, 104, 122, 123, 148] and North America (5.2-6.6) [115, 151, 165]. The lowest DLQI mean scores were reported in Italy (1.8 [89] and 4.3 [90]), Singapore (4.0 [69] and 4.4 [68]), and Nepal (4.1 [38]), whereas the highest DLQI mean scores were reported in Saudi Arabia (14.7 [45] and 9.0 [64]) and Egypt (13.0 [144], 12.4 [87], 12.2 [25], and 11.2-11.9 [19]). Factors affecting DLQI scores were not examined.

In contrast, the Vitiligo-specific QoL (VitiQoL) instrument has only been reported in eight studies [13, 16, 29,
Fig. 1 Preferred Reporting Items for Systematic Reviews and Meta-Analyses (PRISMA) flow diagram
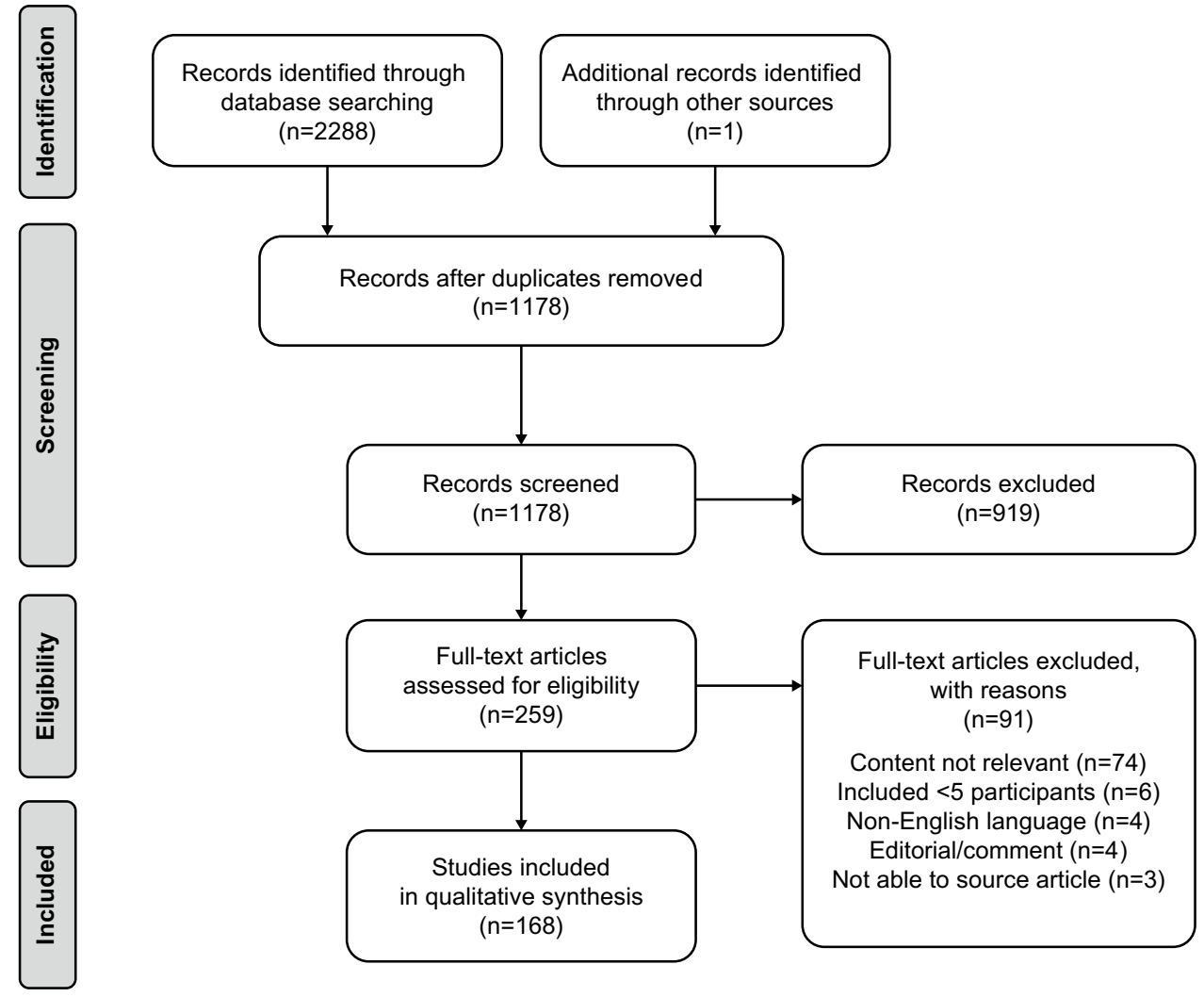
Table 1 Summary of study characteristics

\begin{tabular}{|c|c|}
\hline Characteristic & $\begin{array}{l}\text { Number of } \\
\text { studies, } n(\%) \\
N=168\end{array}$ \\
\hline \multicolumn{2}{|l|}{ Year of publication } \\
\hline 1979-1999 & $13(7.7)$ \\
\hline 2000-2009 & $33(19.6)$ \\
\hline 2010-2021 & $122(72.6)$ \\
\hline \multicolumn{2}{|l|}{ Study type } \\
\hline Observational & $162(96.4)$ \\
\hline Clinical trial & $6(3.6)$ \\
\hline \multicolumn{2}{|l|}{ Geographic region $^{\mathrm{a}}$} \\
\hline Africa & $4(2.4)$ \\
\hline Europe & $48(28.6)$ \\
\hline Eastern Asia ${ }^{\mathrm{b}}$ & $18(10.7)$ \\
\hline Southern Asia & $26(15.5)$ \\
\hline Middle East & $50(29.8)$ \\
\hline North America & $20(11.9)$ \\
\hline South America & $6(3.6)$ \\
\hline \multicolumn{2}{|c|}{ Age group of patients with vitiligo, years ${ }^{c}$} \\
\hline Child only $(<12)$ & $1(0.6)$ \\
\hline Adolescent only (12-17) & $1(0.6)$ \\
\hline Adult only $(\geq 18)$ & $87(51.8)$ \\
\hline Child and adolescent $(\leq 17)$ & $13(7.7)$ \\
\hline Adolescent and adult $(\geq 12)$ & $42(25.0)$ \\
\hline All age groups $(\geq 0)$ & $15(8.9)$ \\
\hline \multicolumn{2}{|l|}{ Number of patients with vitiligo ${ }^{\mathrm{d}}$} \\
\hline$\leq 25$ & $15(8.9)$ \\
\hline $26-100$ & $77(45.8)$ \\
\hline $101-200$ & $38(22.6)$ \\
\hline$>200$ & 30 (17.9) \\
\hline
\end{tabular}

${ }^{a}$ Multinational studies conducted in 2 geographic regions are listed under both regions

${ }^{\mathrm{b}}$ Includes East (Northeast) Asia and Southeast Asia

${ }^{c}$ Patient age groups were not reported for 9 (5.4\%) studies

${ }^{\mathrm{d}}$ The number of patients with vitiligo was not available for 8 studies, which reported on the perceptions of others toward patients with vitiligo $(n=5)$ and caregiver burden $(n=3)$

$32,67,80,88,115]$ using our search parameters; half were published in the past year, of which two were clinical trials. There were no notable regional differences in VitiQoL scores among studies, which were conducted in each of the seven geographic regions, with two in North America. Another instrument specific to vitiligo, the Vitiligo Impact Scale (VIS), was only used in three studies, with two published in the past year. One study used the original 27-item VIS [32], and two used the abbreviated 22-item scale (VIS22) [36, 100]; all studies were conducted in Southern Asian populations. Although the use of vitiligo-specific scales has increased recently, there remains an unmet need for a widely utilized, vitiligo-specific QoL instrument that has been validated in large interventional studies.

\subsection{Psychosocial Comorbidities}

\subsubsection{Prevalence of Psychosocial Comorbidities}

A summary of studies that reported psychosocial comorbidities is presented in Table 2 (complete information presented in Table 1 of the ESM). Nine studies noted the presence of any (unspecified) psychosocial comorbidity in 32.6-90.0\% of patients with vitiligo $[22,23,34,39,42-44,138,145$, 173]. Depression and anxiety were the most commonly reported psychosocial comorbidities. Forty-one studies reported depression or depressive disorders (including major depressive disorder, bipolar disorder, and dysthymic disorder) in patients with vitiligo, with a prevalence range from $0.1-62.3 \%[15,17,22,23,28,30,32,34,35,39,40,43,44$, $46,48,50,57,68,69,71,92,100,104,113-115,131,137$, $138,142,143,145,146,150,154,155,158-160,163,177]$. Twenty studies reported anxiety or anxiety-related disorders (including generalized anxiety disorder, agoraphobia, social phobia [not social avoidance], and panic disorder), with a prevalence of $1.9-67.9 \%[22,23,30,34,39,43,44,46$, 50, 57, 92, 115, 137, 138, 150, 154, 155, 158, 159, 175]. Among studies that used the same rating scales for determining the prevalence of depression or anxiety, ranges were more narrow (Table 2). Concomitant depression and anxiety was reported in four studies (4.9-33.3\%) [34, 43, 44, 159].

Other psychosocial comorbidities were also widely reported and included feelings of stigmatization (eight studies, 17.3-100\%) [32, 101, 102, 131-133, 136, 169], sleep disturbance (seven studies, 4.6-89.0\%) [92, 109, 117, 143, $150,154,167]$, alexithymia (four studies, 23.8-46.7\%) [72, $108,129,142]$, anger (six studies, 14-36.9\%) [40, 46, 141, $142,145,158]$, and somatoform disorder (three studies, 6.3-9.4\%) [22, 137, 150]. Various impairments were noted, including emotional impairment (11 studies, 6-65.0\%) [13, 23, 32, 40, 46, 56, 131-133, 141, 145]; cognitive impairment (three studies, 0.3-50.8\%) [23, 137, 145]; and behavioral impairments that included avoidance or restriction behavior (nine studies, 12.5-76\%) [32, 57, 61, 100, 102, 109, 130, 141, 146], attention-deficit/hyperactivity disorder (one study, 20.0\%) [34], obsessive disorders (five studies, $0.1-19.5 \%)[23,39,137,138,154]$, and binge-eating disorder (one study, 7.4\%) [22]. Alcohol dependence or abuse was reported in three studies $(2.4-7.6 \%)$ [22, 57, 137]. Patients were affected by adjustment disorders, such as stress associated with vitiligo and worry about spread (12 studies, 4-93.9\%) [13, 30, 32, 40, 100, 109, 113, 114, 131, 141, $142,149]$, and also experienced aspects of self-consciousness, including embarrassment (eight studies, 24-66.7\%) $[13,32,131-133,135,142,145]$ and low self-esteem (four 


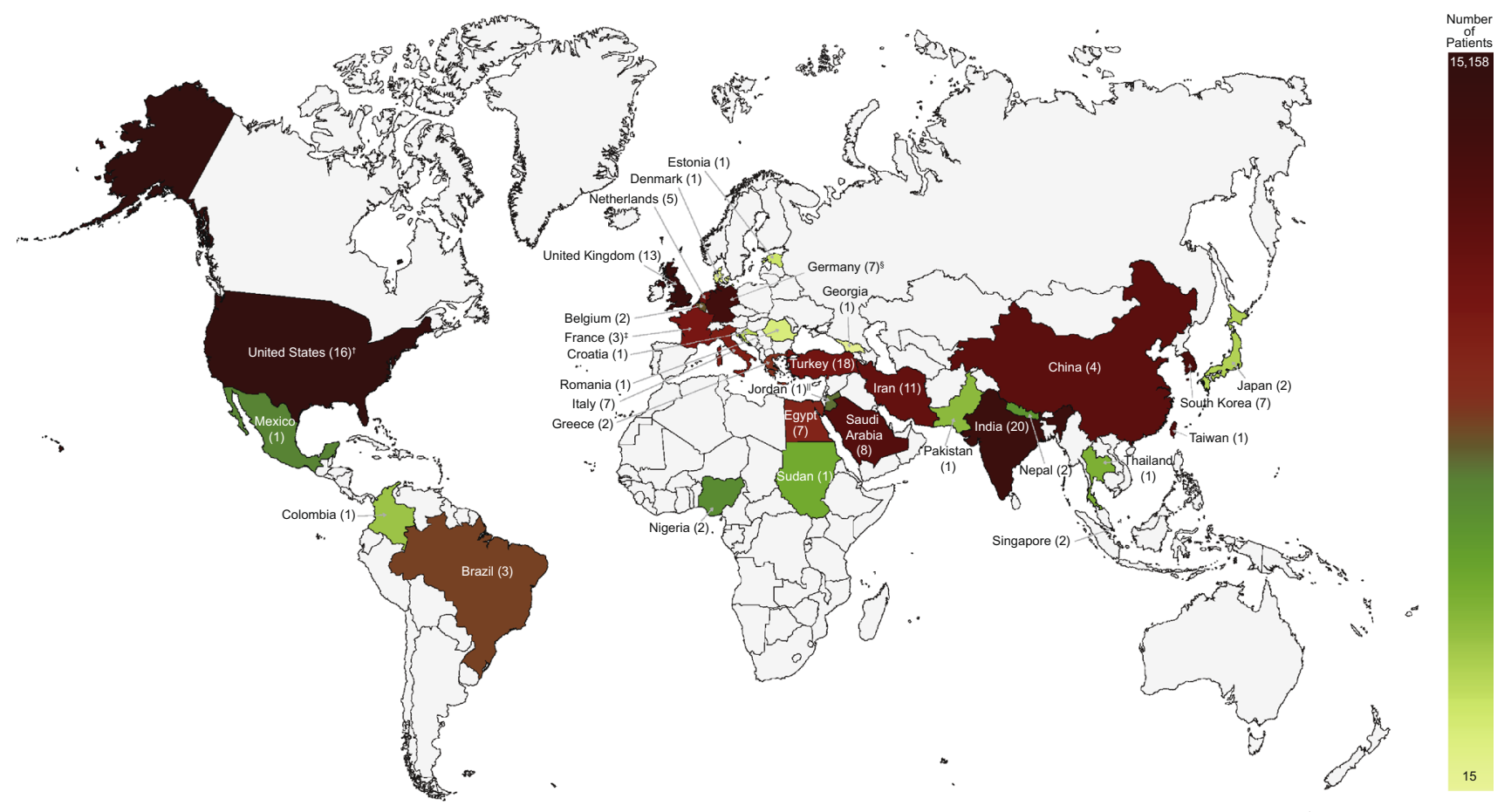

Fig. 2 Number of patients with vitiligo in included studies by country and number of studies. * Number of patients is the sum of patients across studies from each country with multiple populations from the same patient population excluded; the number of unique studies is shown in parentheses after the name of each country. ${ }^{\dagger}$ Includes three studies with populations in the USA and a European country (France, one study [ $n=442]$; Germany, two studies [ $n=85$ and $n=74]$ ). Within each study, the number of patients in each country was not available in the published studies; thus, the full population is included in both countries on this map. ${ }^{\ddagger}$ Includes one study with a population in France and the USA $(n=442)$. The number of patients in each

studies, 6.2-72.7\%) [32, 68, 69, 141]. Relationship difficulties including sexual dysfunction were reported over a wide range of patients (ten studies, 2.0-81.8\%) [32, 43, 97, $109,131,132,135,154,155,167]$. Suicidality was reported among patients with vitiligo, including unspecified suicidality (one study, 28.3\%) [138], suicidal ideation (six studies, 3-25.0\%) [32, 100, 131, 143, 150, 154], and suicide attempts (two studies, 3.3-3.7\%) [150, 154].

\subsubsection{Degree of Psychosocial Burden}

Compared with controls, psychosocial comorbidities noted to be significantly $(p \leq 0.05)$ associated with vitiligo were depression (11 studies) [23, 28, 33, 34, 55, 63, 128, 140, $143,145,155]$, anxiety (ten studies) [23, 30, 33, 34, 55, $63,119,128,140,155]$, emotional or behavioral impairment (six studies) $[9,23,34,128,140,161]$, adjustment disorder (four studies) [15, 30, 37, 128], low self-esteem country was not available in the published study; thus, the full population is included in both countries on this map. ${ }^{\S}$ Includes two studies with populations in Germany and the USA ( $n=85$ and $n=74$ ) and one study with a population in Germany and Jordan $(n=167)$. Within each study, the number of patients in each country was not available in the published studies; thus, the full population is included in both countries on this map. "Includes one study with a population in Jordan and Germany $(n=167)$. The number of patients in each country was not available in the published study; thus, the full population is included in both countries on this map

(three studies) [57, 65, 134], relationship and sexual dysfunction (three studies) $[33,155,161]$, sleep disturbance (two studies) [55, 117], suicidality (one study) [128], self-consciousness (one study) [119], embarrassment (one study) [145], alexithymia (one study) [108], and alcohol abuse or addiction (one study) [128]. Five studies reported that depression $[30,84,85,102,164]$ and/or anxiety [84, 164] scores measured by Beck Inventory scales were not significantly different in patients with vitiligo vs controls.

The QoL and/or psychosocial burden of vitiligo was most frequently compared with psoriasis (26 articles) [4, $9,27,58,60,76,83,84,87,92,110,111,113,118,123$, 130, 134, 136, 137, 150, 154, 159, 162, 173, 174], alopecia areata (13 articles) $[14,24,58,60,75,76,83,109,118$, $154,159,173,174]$, acne (11 articles) [14, 58, 76, 117, $118,137,140,154,159,174,175]$, atopic dermatitis (ten articles) [27, 58, 73, 76, 110, 111, 119, 154, 173, 174], and urticaria (nine articles) $[27,58,76,137,154,155$, 173-175]. Table 3 lists the prevalence of psychosocial 
Table 2 Prevalence of psychosocial comorbidity in patients with vitiligo

\begin{tabular}{lll}
\hline Psychosocial comorbidity Comorbidity screening tool & $\begin{array}{l}\text { Number of } \\
\text { patients with } \\
\text { vitiligo }\end{array}$ & Prevalence, \% Country
\end{tabular}

Depression or depressive disorders Any

Depression

Bipolar disorder
Dysthymic disorder

MDD

Unspecified depressive disorder

Anxiety or anxiety-related disorders
BDI

CES-D

HADS

PHQ-9

Other $^{\mathrm{a}}$

Diagnosed during screening

Diagnosis on file

Diagnosed during screening

SCID-I

Other $^{\mathrm{a}}$

K-SADS-PL

Any
6-7104

100-308

54-222

15-102

6-104

22-3962

53

1432

113

42-50

42-7104

30

15-1432

100-150

15-102

60.0-66.0

HADS

Other $^{\mathrm{a}}$

Agoraphobia

GAD

Panic disorder

Social phobia

Depression and anxiety
$0.1-62.3$

7.6

0.1

0.9-1.8

4.8-26.0

$3.5-56.6$

23.4

1.9-67.9
PAS

Other $^{\mathrm{a}}$

Other ${ }^{\mathrm{a}}$

Other $^{\mathrm{a}}$

Any

HADS

Other $^{\mathrm{a}}$
30-1432

100

$30-42$

53-95

42-181

15-102

15-102

30-100

Egypt [154], Estonia [92], Georgia [159], Germany [104], India [22, 28, 43, 100, 113, 114, 137, 138, $143,145,146,150,177]$, Italy [142], Iran [30], Japan [163], Jordan [104], South Korea [71], Mexico [115], Nepal [32], Nigeria [44], Saudi Arabia [17, 46, 48, 50], Singapore [68, 69], Taiwan [23], Thailand [35], Turkey [34, 39, 57, $155,158]$, UK [40, 160], USA [15, 131]

Germany [104], India [146], Iran [30], Jordan [104], Mexico [115], Saudi Arabia [17, 48]

Japan [163], South Korea [71], Singapore [68, 69]

Georgia [159], Nigeria [44], Saudi Arabia [50]

Thailand [35], India [177]

Egypt [154], Estonia [92], India [22, 28, 100, 113, 114, 137, 143, 145, 150], Italy [175], Nepal [32], Saudi Arabia [46], Turkey [39, 158], UK [40], USA [15, 131]

India [137]

Taiwan [23]

India $[113,114]$

Turkey [57, 155]

India [22, 43, 138], Taiwan [23], Turkey [57], UK [160]

Turkey [34]

Egypt [154], Estonia [92], Georgia [159], India [22, 43, 137, 138, 150], Italy [175], Iran [30], Mexico [115], Nigeria [44], Saudi Arabia [46, 50], Taiwan [23], Turkey [34, $39,57,155,158]$

Iran [30], Mexico [115]

Georgia [159], Nigeria [44], Saudi Arabia [50]

Egypt [154], Estonia [92], India [22, 43, 137, 150], Saudi Arabia [46], Taiwan [23], Turkey [39, 155, 158] India [43]

Turkey [34, 57]

Estonia [92], India [22, 138]

Estonia [92], India [43, 138], Italy [175], Turkey [57]

Georgia [159], India [43], Nigeria [44], Turkey [34]

Georgia [159], Nigeria [44]

India [43], Turkey [34] 
Table 2 (continued)

\begin{tabular}{|c|c|c|c|c|}
\hline Psychosocial comorbidity & Comorbidity screening tool & $\begin{array}{l}\text { Number of } \\
\text { patients with } \\
\text { vitiligo }\end{array}$ & Prevalence, $\%$ & Country \\
\hline Stigmatization & Other $^{\mathrm{a}}$ & $7-326$ & $17.3-100$ & $\begin{array}{l}\text { Germany [101, 102], India [136], } \\
\text { Nepal [32], UK [169], USA [101, } \\
\text { 131, 132] }\end{array}$ \\
\hline Adjustment disorders & Any & $22-326$ & 4-93.9 & $\begin{array}{l}\text { Egypt [149], Germany [141], Italy } \\
\text { [142], India [100, 113, 114], Iran } \\
\text { [30], Nepal [32], Nigeria [13], } \\
\text { Romania [109], UK [40], USA } \\
\text { [131] }\end{array}$ \\
\hline Adjustment disorder & Diagnosed during screening & 113 & $10.6-11.5$ & India $[113,114]$ \\
\hline Helplessness & VIS & 22 & 9.1 & Nepal [32] \\
\hline Hopelessness & BHS & 100 & 60.0 & Iran [30] \\
\hline \multirow[t]{2}{*}{ Stress } & $\begin{array}{l}\text { Holmes and Rahe Social Read- } \\
\text { justment Rating Scale }\end{array}$ & $30-32$ & $65.6-93.9$ & Egypt [149], Romania [109] \\
\hline & Freiburger Personality Inventory & 117 & 28.2 & Germany [141] \\
\hline Unhappiness & Self-report & 22 & 68.2 & Nepal [32] \\
\hline Worry about others' thoughts & VitiQoL & $22-29$ & $31.8-40.9$ & Nepal [32], Nigeria [13] \\
\hline \multirow[t]{2}{*}{ Worry about spread } & VitiQoL & $22-29$ & $68.2-75.9$ & Nepal [32], Nigeria [13] \\
\hline & Other $^{\mathrm{a}}$ & $22-326$ & $4-88.0$ & $\begin{array}{l}\text { India [100], Italy [142], Nepal [32], } \\
\text { UK [40], USA [131] }\end{array}$ \\
\hline Sleep disturbances & Other $^{\mathrm{a}}$ & $30-116$ & $4.6-89.0$ & $\begin{array}{l}\text { Egypt [154], Estonia [92], Greece } \\
\text { [117], India [143, 150], Romania } \\
\text { [109], UK [167] }\end{array}$ \\
\hline Behavioral impairment & Any & $22-1432$ & $0.1-76$ & $\begin{array}{l}\text { Egypt [154], France [61], Germany } \\
\text { [102, 141], India [22, 100, 130, } \\
\text { 137, 138, 146], Nepal [32], Roma- } \\
\text { nia [109], Taiwan [23], Turkey [34, } \\
\text { 39, 57], USA [61] }\end{array}$ \\
\hline ADHD & K-SADS-PL & 30 & 20.0 & Turkey [34] \\
\hline Binge-eating disorder & PRIME-MD PHQ & 95 & 7.4 & India [22] \\
\hline \multirow[t]{3}{*}{ Obsessive disorders } & Diagnosed during screening & $53-113$ & $7.6-19.5$ & Egypt [154], India [137], Turkey [39] \\
\hline & Diagnosis on file & 1432 & 0.1 & Taiwan [23] \\
\hline & Not specified & 53 & 3.8 & India [138] \\
\hline \multirow{2}{*}{$\begin{array}{l}\text { Social and situational avoidance/ } \\
\text { restriction }\end{array}$} & Participation Scale & $100-150$ & $17.3-48.0$ & India $[130,146]$ \\
\hline & Other $^{\mathrm{a}}$ & $22-442$ & $12.5-76$ & $\begin{array}{l}\text { France [61], Germany [102, 141], } \\
\text { India [100], Nepal [32], Romania } \\
\text { [109], Turkey [57], USA [61] }\end{array}$ \\
\hline Self-consciousness & Any & $22-326$ & $6.2-72.7$ & $\begin{array}{l}\text { Germany [141], India [145], Italy } \\
\text { [142], Nepal [32], Nigeria [13], } \\
\text { Singapore [68, 69], USA [131-133, } \\
\text { 135] }\end{array}$ \\
\hline \multirow[t]{2}{*}{ Embarrassment } & VitiQoL & $22-29$ & $27.3-55.5$ & Nepal [32], Nigeria [13] \\
\hline & Other $^{\mathrm{a}}$ & $22-326$ & $24-66.7$ & $\begin{array}{l}\text { India [145], Italy [142], Nepal [32], } \\
\text { USA [131-133, 135] }\end{array}$ \\
\hline \multirow[t]{2}{*}{ Low self-esteem } & RSES & $145-222$ & $6.2-6.8$ & Singapore $[68,69]$ \\
\hline & Other $^{\mathrm{a}}$ & $22-117$ & $30-72.7$ & Germany [141], Nepal [32] \\
\hline \multirow[t]{3}{*}{ Emotional impairment } & Any & $22-1432$ & $6-65.0$ & $\begin{array}{l}\text { Germany [141], India [145], Nepal } \\
\text { [32], Nigeria [13], South Korea } \\
\text { [56], Saudi Arabia [46], Taiwan } \\
\text { [23], UK [40], USA [131-133] }\end{array}$ \\
\hline & VitiQoL & $22-29$ & $18.2-55.2$ & Nepal [32], Nigeria [13] \\
\hline & Other $^{\mathrm{a}}$ & $61-1432$ & $6-65.0$ & $\begin{array}{l}\text { Germany [141], India [145], South } \\
\text { Korea [56], Saudi Arabia [46], Tai- } \\
\text { wan [23], UK [40], USA [131-133] }\end{array}$ \\
\hline
\end{tabular}


Table 2 (continued)

\begin{tabular}{|c|c|c|c|c|}
\hline Psychosocial comorbidity & Comorbidity screening tool & $\begin{array}{l}\text { Number of } \\
\text { patients with } \\
\text { vitiligo }\end{array}$ & Prevalence, $\%$ & Country \\
\hline Cognitive impairment & Any & $53-1432$ & $0.3-50.8$ & India [137, 145], Taiwan [23] \\
\hline \multirow[t]{2}{*}{ Schizophrenia } & Diagnosed during screening & 53 & 3.8 & India [137] \\
\hline & Diagnosis on file & 1432 & 0.3 & Taiwan [23] \\
\hline Unspecified & Skindex-61 & 61 & 50.8 & India [145] \\
\hline $\begin{array}{l}\text { Relationship difficulties and sexual } \\
\text { dysfunction }\end{array}$ & Any & $22-326$ & $2.0-81.8$ & $\begin{array}{l}\text { Egypt [154], India [43], Nepal [32], } \\
\text { South Korea [97], Romania [109], } \\
\text { Turkey [155], UK [167], USA [131, } \\
132,135]\end{array}$ \\
\hline Relationships & Other $^{\mathrm{a}}$ & $22-326$ & $4.5-81.8$ & Nepal [32], USA [131, 135] \\
\hline Sexual & Other $^{\mathrm{a}}$ & $32-167$ & $2.0-48.2$ & $\begin{array}{l}\text { Egypt [154], India [43], South Korea } \\
\text { [97], Romania [109], Turkey [155], } \\
\text { UK [167], USA [132, 135] }\end{array}$ \\
\hline Alexithymia & TAS-20 & $30-181$ & $23.8-46.7$ & Iran $[72,108]$, Italy $[129,142]$ \\
\hline \multirow[t]{3}{*}{ Anger } & Any & $61-181$ & $14-36.9$ & $\begin{array}{l}\text { Germany [141], India [145], Italy } \\
\text { [142], Saudi Arabia [46], Turkey } \\
\text { [158], UK [40] }\end{array}$ \\
\hline & IPQ & $100-164$ & $29.0-34$ & Saudi Arabia [46], Turkey [158] \\
\hline & Other $^{\mathrm{a}}$ & $61-181$ & $14-36.9$ & $\begin{array}{l}\text { Germany [141], India [145], Italy } \\
\text { [142], UK [40] }\end{array}$ \\
\hline Suicidality & Any & $30-326$ & $3-28.3$ & $\begin{array}{l}\text { Egypt [154], India [100, 138, 143, } \\
\text { 150], USA [131] }\end{array}$ \\
\hline Attempts & Diagnosed during screening & $30-108$ & $3.3-3.7$ & Egypt [154], India [150] \\
\hline Ideation & Other $^{\mathrm{a}}$ & $22-326$ & $3-25.0$ & $\begin{array}{l}\text { Egypt [154], India [100, 143, 150], } \\
\text { Nepal [32], USA [131] }\end{array}$ \\
\hline Unspecified & Not specified & 53 & 28.3 & India [138] \\
\hline Somatoform disorder & Other ${ }^{\mathrm{a}}$ & $30-95$ & $6.3-9.4$ & India $[22,137,150]$ \\
\hline Alcohol dependence or abuse & Other $^{\mathrm{a}}$ & $42-95$ & $2.4-7.6$ & India $[22,137]$, Turkey [57] \\
\hline
\end{tabular}

$A C S$ Adjustment to Chronic Skin Disorders Questionnaire, $A D H D$ attention-deficit/hyperactivity disorder, ASEX Arizona Sexual Experience Scale, BAI Beck Anxiety Inventory, BDI Beck Depression Inventory, BHS Beck Hopelessness Scale, CES-D Center for Epidemiologic Studies Depression Scale, DLQI Dermatology Life Quality Index, ES-Q Emotional State Questionnaire, GAD generalized anxiety disorder, GHQ General Health Questionnaire, GHQ-H Hindi version of the General Health Questionnaire, HADS Hospital Anxiety and Depression Scale, HDRS Hamilton Depression Rating Scale, IPQ Illness Perception Questionnaire, $K$-SADS-PL Schedule for Affective Disorders and Schizophrenia for School Age Children-Present and Lifetime Version, $M D D$ major depressive disorder, $P A S$ Psychiatric Assessment Schedule, $P H Q-9$ Patient Health Questionnaire-9, PRIME-MD PHQ Primary Care Evaluation of Mental Disorders-Patient Health Questionnaire, QIDS-SR-16 Quick Inventory of Depressive Symptomatology-Self Report, RSES Rosenberg Self-Esteem Scale, SCID-I Structured Clinical Interview for DSMIV Axis I Disorders, SDS Sheehan Disability Scale, SRE Schedule of Recent Experience, TAS-20 Toronto Alexithymia Scale-20, VIS Vitiligo Impact Scale, VIS-22 Vitiligo Impact Scale 22, VitiQoL Vitiligo-specific Quality of Life

${ }^{a}$ Reporting based on diagnosis or self-report, an unspecified tool, or a tool used in only 1 study for each comorbidity; if a comorbidity only included data from 1 study, the specific tool (including diagnosis or self-report) was listed. For "diagnosis on file" and "diagnosed during screening," formal diagnosis or diagnostic criteria/codes (i.e., Diagnostic and Statistical Manual of Mental Disorders IV/V or International Classification of Diseases 9/10) suggestive of a formal diagnosis were provided in the article. Tools used in only 1 study per comorbidity include ACS (stigmatization, social and situational avoidance/restriction), ASEX (sexual dysfunction), DLQI (sleep disturbances), ES-Q (depression, anxiety, panic disorder, social phobia, sleep disturbances), Freiburger Personality Inventory (social and situational avoidance/restriction, low self-esteem, emotional impairment, anger), GHQ-H (sleep disturbances, somatoform disorder), HDRS (depression, MDD, suicidal ideation), Holmes and Rahe Social Readjustment Rating Scale (sleep disturbances, social and situational avoidance/restriction, sexual dysfunction), IPQ (depression, emotional impairment), K-SADS-PL (GAD, depression and anxiety), Participation Scale (stigmatization), PAS (MDD, anxiety, social phobia, depression and anxiety, sexual dysfunction), PRIME-MD PHQ (depression, MDD, anxiety, panic disorder, somatoform disorder, alcohol dependence or abuse), QIDS-SR-16 (depression, suicidal ideation), SCID-I (MDD, anxiety, GAD, social phobia, alcohol dependence or abuse), SDS (social and situational avoidance/restriction), Skindex-29 (depression, social phobia, worry about spread, embarrassment, emotional impairment, anger), Skindex-61 (depression, embarrassment, emotional impairment, anger), SRE (sleep disturbances, sexual dysfunction), VIS (depression, worry about spread, social and situational avoidance/restriction, embarrassment, relationship difficulties, suicidal ideation), VIS-22 (worry about spread, social and situational avoidance/restriction), and VitiQoL (social and situational avoidance/restriction). Additional details are available in Table 1 of the ESM 
Table 3 Psychosocial comorbidity burden in patients with vitiligo compared with other skin diseases

\begin{tabular}{|c|c|c|c|c|c|c|}
\hline $\begin{array}{l}\text { Psychosocial } \\
\text { comorbidity, \% }\end{array}$ & Vitiligo vs acne & $\begin{array}{l}\text { Vitiligo vs alopecia } \\
\text { areata }\end{array}$ & $\begin{array}{l}\text { Vitiligo vs atopic } \\
\text { dermatitis }\end{array}$ & Vitiligo vs psoriasis & Vitiligo vs urticaria & Vitiligo vs eczema \\
\hline Depression & $\begin{array}{l}\mathbf{4 6 . 3} \text { vs } 18.9[154] \\
\mathbf{4 7 . 2} \text { vs } \mathbf{1 9 . 6}[137] \\
\mathbf{6 0 . 0} \text { vs } \mathbf{5 4 . 1}[159]\end{array}$ & $\begin{array}{l}46.3 \text { vs } 55.3[154] \\
\mathbf{6 0 . 0} \text { vs } \mathbf{5 0 . 0}[159]\end{array}$ & 46.3 vs $35.5[154]$ & $\begin{array}{l}\mathbf{4 6 . 3} \text { vs } \mathbf{4 2 . 3}[154] \\
\mathbf{4 7 . 2} \text { vs } \mathbf{3 4 . 8}[137] \\
60.0 \text { vs } 69.4[159] \\
10.0 \text { vs } 23.3[150] \\
4.4 \text { vs } 6.8[113]\end{array}$ & $\begin{array}{l}\mathbf{4 6 . 3} \text { vs } 35.5[154] \\
\mathbf{4 7 . 2} \text { vs } \mathbf{4 3 . 6}[137]\end{array}$ & $\mathbf{6 0 . 0}$ vs 56.6 [159] \\
\hline Anxiety & $\begin{array}{l}31.5 \text { vs } 55.3 \text { [154] } \\
11.3 \text { vs } 24.7 \text { [137] } \\
66.7 \text { vs } 78.4 \text { [159] }\end{array}$ & $\begin{array}{l}\mathbf{3 1 . 5} \text { vs } 19.7[154] \\
\mathbf{6 6 . 7} \text { vs } 64.3[159]\end{array}$ & $31.5 \mathrm{vs} 43.6[154]$ & $\begin{array}{l}\mathbf{3 1 . 5} \text { vs } \mathbf{2 4 . 3}[154] \\
11.3 \text { vs } 13.5[137] \\
66.7 \text { vs } 83.3[159] \\
3.3 \text { vs } 3.3[150]\end{array}$ & $\begin{array}{l}31.5 \text { vs } 43.6[154] \\
11.3 \text { vs } 11.5[137] \\
6.0 \text { vs } 30.0[155] \\
(p=0.003)\end{array}$ & 66.7 vs 73.3 [159] \\
\hline $\begin{array}{l}\text { Depression and } \\
\text { anxiety }\end{array}$ & 33.3 vs 21.6 [159] & 33.3 vs 21.4 [159] & & 33.3 vs 38.8 [159] & & 33.3 vs 26.7 [159] \\
\hline $\begin{array}{l}\text { Sleep distur- } \\
\text { bances }\end{array}$ & 4.6 vs $1.9[154]$ & 4.6 vs $2.4[154]$ & 4.6 vs 51.8 [154] & $\begin{array}{l}4.6 \text { vs } 22.0[154] \\
20.0 \text { vs } 56.7[150]\end{array}$ & 4.6 vs 51.8 [154] & \\
\hline Suicide & & & & & & \\
\hline Ideation & 25.0 vs 11.7 [154] & 25.0 vs 38.5 [154] & $\mathbf{2 5 . 0}$ vs 19.1 [154] & $\begin{array}{l}\mathbf{2 5 . 0} \text { vs } \mathbf{1 0 . 0}[154] \\
10.0 \text { vs } 26.6[150]\end{array}$ & 25.0 vs 19.1 [154] & \\
\hline Attempt & 3.7 vs 2.4 [154] & 3.7 vs 4.3 [154] & 3.7 vs $0[154]$ & 3.7 vs $4.3[154]$ & 3.7 vs 0 [154] & \\
\hline Sexual dysfunction & 48.2 vs $6.8[154]$ & 48.2 vs $20.2[154]$ & 48.2 vs 21.8 [154] & $\mathbf{4 8 . 2}$ vs $40.0[154]$ & $\begin{array}{l}\mathbf{4 8 . 2} \text { vs } \mathbf{2 1 . 8}[154] \\
36.0 \text { vs } 58.0[155]\end{array}$ & \\
\hline $\begin{array}{l}\text { Somatoform } \\
\text { disorder }\end{array}$ & 9.4 vs 8.3 [137] & & & 9.4 vs $5.6[137]$ & 9.4 vs 9.0 [137] & \\
\hline $\begin{array}{l}\text { Adjustment } \\
\text { disorder }\end{array}$ & O vs 10.3 [137] & & & $\begin{array}{l}0 \text { vs } 4.5[137] \\
11.5 \text { vs } 14.6[113]\end{array}$ & $\begin{array}{l}\text { O vs } 7.7 \\
{[137]}\end{array}$ & \\
\hline $\begin{array}{l}\text { Obsessive-com- } \\
\text { pulsive disorder }\end{array}$ & $\begin{array}{l}\mathbf{1 8 . 5} \text { vs } \mathbf{3 . 9}[154] \\
7.6 \text { vs } 11.3[137]\end{array}$ & 18.5 vs 2.9 [154] & 18.5 vs 2.7 [154] & $\begin{array}{l}\mathbf{1 8 . 5} \text { vs } 2.7[154] \\
\mathbf{7 . 6} \text { vs } 2.3[137]\end{array}$ & $\begin{array}{l}\mathbf{1 8 . 5} \text { vs } 2.7[154] \\
\mathbf{7 . 6} \text { vs } 2.6[137]\end{array}$ & \\
\hline $\begin{array}{l}\text { Bipolar affective } \\
\text { disorder }\end{array}$ & 7.6 vs 2.1 [137] & & & $\mathbf{7 . 6}$ vs 3.4 [137] & 7.6 vs 3.9 [137] & \\
\hline Schizophrenia & 3.8 vs 3.1 [137] & & & 3.8 vs 5.6 [137] & 3.8 vs 2.6 [137] & \\
\hline $\begin{array}{l}\text { Alcohol depend- } \\
\text { ence syndrome }\end{array}$ & 7.6 vs 2.1 [137] & & & 7.6 vs 11.2 [137] & 7.6 vs 5.1 [137] & \\
\hline Dysthymia & & & & 1.8 vs $1.0[113]$ & $\begin{array}{l}26.0 v s 46.0[155] \\
(p=0.04)\end{array}$ & \\
\hline Stigma & & & & 17.3 vs 28.0 [136] & & \\
\hline Stress $^{\mathrm{a}}$ & & 65.6 vs 68.9 [109] & & & & \\
\hline $\begin{array}{l}\text { Participation } \\
\text { restriction }\end{array}$ & & & & 17.3 vs 28.0 [130] & & \\
\hline $\begin{array}{l}\text { Unspecified } \\
\text { psychiatric } \\
\text { comorbidity }\end{array}$ & & $\mathbf{7 9 . 2}$ vs $60.0[173]$ & 79.2 vs 56.3 [173] & $\begin{array}{l}79.2 \text { vs } 80.0[173] \\
16.7 \text { vs } 53.3[150] \\
(p=0.003)\end{array}$ & 79.2 vs $70.6[173]$ & \\
\hline
\end{tabular}

Bolded cells indicate a higher prevalence in vitiligo; italics cells indicate a lower prevalence in vitiligo

${ }^{a}$ Stress includes stressful events that stem from family, personal, work, and financial problems 


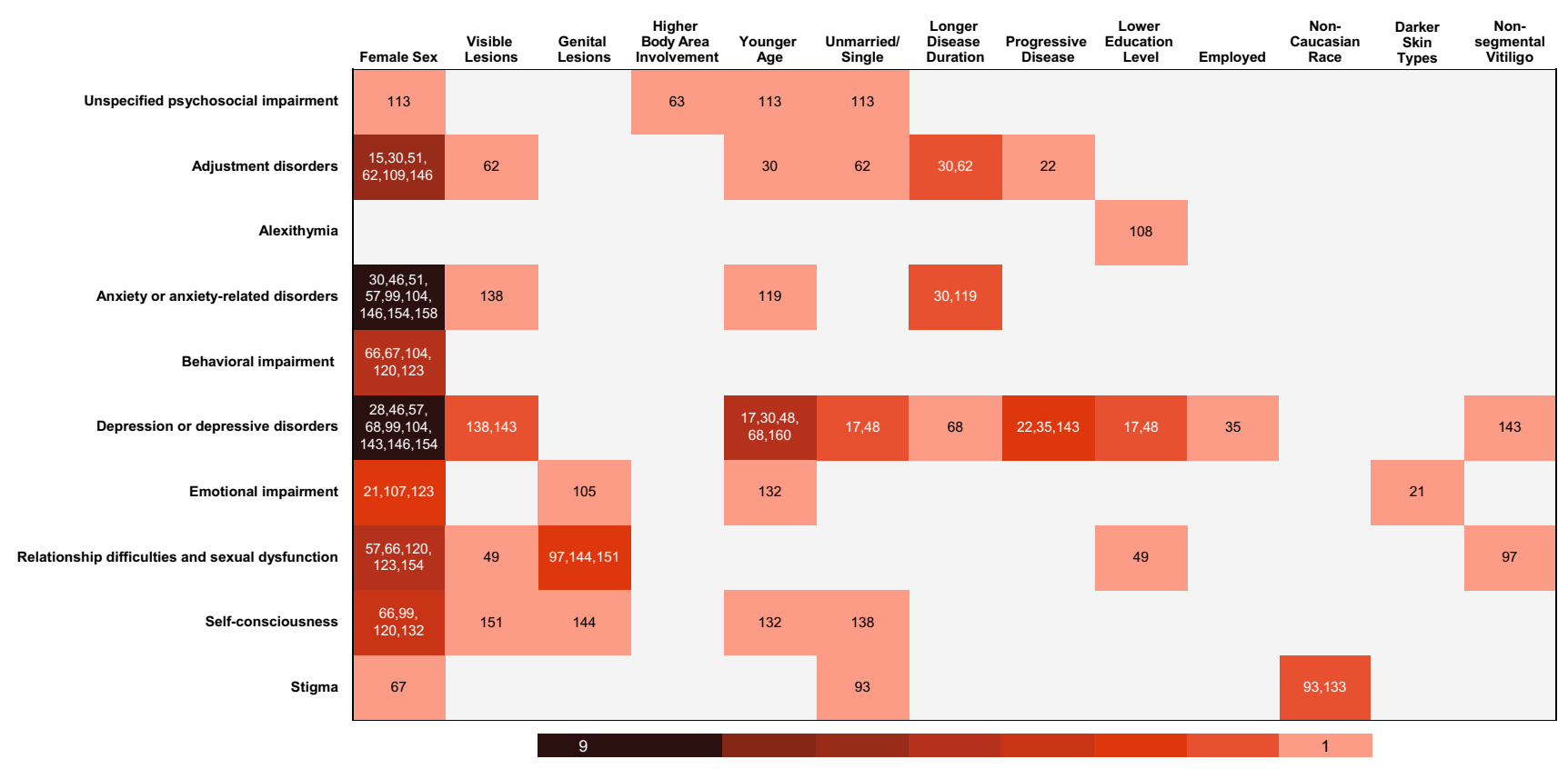

Fig. 3 Heat map showing the references that report factors significantly associated with psychosocial comorbidity. Significance was conferred at $p \leq 0.05$. Darker red shading indicates a larger number of studies reporting significant associations

comorbidity in vitiligo compared with other skin diseases. In general, psychosocial comorbidities were more prevalent in vitiligo compared with acne, alopecia areata, atopic dermatitis, and urticaria but less prevalent vs psoriasis. Regarding non-dermatologic diseases, one study reported comparable SF-36 mental component scores in patients with vitiligo vs chronic lung disease, arthritis, cancer, and congestive heart failure [9].

\subsubsection{Factors Associated with Psychosocial Burden}

Factors that were significantly associated with higher psychosocial (Fig. 3) or overall QoL burden were female sex (30 studies) $[15,21,25,28,30,45,46,49,51,57,62,66-68,70$, $88,90,99,104,107,109,112,113,120,123,132,143,146$, $154,158]$, lesion location in visible areas (e.g., face, hands [17 studies]) [4, 25, 35, 37, 49, 62, 70, 80, 89, 90, 92, 101, $122,123,138,143,151]$ or genitals (eight studies) [70, 89, $97,105,115,122,144,151]$, younger age (16 studies; particularly those aged $<30$ years and more so in adolescents) $[17,25,30,48,49,62,67,68,70,88,100,113,119,132$, $151,160]$, and extensive body area involvement (13 studies) $[4,25,63,65,70,89,92,96,98,107,151,152,166]$. Unmarried and/or single relationship status (nine studies) $[17,22,48,49,62,70,93,113,138]$, longer disease duration (nine studies; particularly duration $>5$ years) $[4,25,30,62$, $65,68,88,107,119]$, progressive disease (seven studies) [22, 25, 35, 89, 92, 107, 143], Fitzpatrick skin phototype
IV-VI (five studies) [21, 35, 37, 77, 107], lower education status (five studies; particularly high school or lower level of education) $[17,48,49,108,136]$, non-segmental vitiligo (three studies; vs segmental or focal vitiligo) [97, 115, 143], non-Caucasian race (three studies) [93, 96, 133], positive family history of vitiligo (two studies) [98, 101], being employed (one study; compared with being students, unemployed, or retired) [35], and higher socioeconomic level (one study) [25] were also significantly associated with increased burden. Four studies reported that comorbid depression significantly reduced overall QoL [35, 92, 100, 115]. Management strategies [107] including camouflage [18, 25, 102, $122,156]$, cognitive behavioral therapy $[26,125,127,148$, 168], phototherapy [51, 52, 157], and depigmentation cream (in patients with extensive vitiligo) [19] were associated with decreased vitiligo-associated burden.

\subsection{Coping Strategies Among Patients with Vitiligo}

The most commonly discussed coping strategies in studies included the use of concealing clothing (six studies, $8.3-78.3 \%$ of patients) $[25,74,101,102,131,157]$, camouflage (four studies, $14.6-62.0 \%$ of patients) [25, 74, 102, 131], and altered body movements (three studies, $5.9-8.1 \%$ of patients) [74, 101, 102]. Other coping strategies included vitiligo acceptance [42, 132, 170], avoidance behavior [101, $102,170]$, and psychotherapy or support groups [42, 61, $170]$. 


\subsection{Perceptions Toward Vitiligo}

Perceptions toward vitiligo were discussed in 13 articles; seven articles focused on perceptions of patients toward their vitiligo [32, 46, 79, 126, 158, 165, 172], and six focused on perceptions of others toward patients with vitiligo [31, 47, $54,78,91,171]$. Several articles covered aspects of knowledge or beliefs about vitiligo, including attitudes and behaviors. Common misperceptions included thinking that vitiligo is contagious [31, 47, 54, 91, 172] and that vitiligo is caused by external forces (e.g., "evil eye," witchcraft/sorcery, evil spirits/Jinn, chance/fate) [31, 32, 46, 47, 79, 158], lack of hygiene $[47,91]$, or infection with germs or viruses $[47,54$, $79,158]$. In three studies that investigated attitudes toward patients with vitiligo, participants with sufficient knowledge of vitiligo vs insufficient knowledge reported a lower prevalence of negative attitudes and a higher prevalence of positive attitudes [31, 78, 91]. In four studies that reported a willingness to have a relationship with or marry someone with vitiligo, $6.7-43.9 \%$ of participants responded in the affirmative [31, 47, 54, 78]; reasons for refusing marriage included social reasons, the impact of vitiligo on appearance, and that vitiligo is believed to be inherited or contagious $[31,47]$.

\subsection{Caregiver Burden}

Caregiver (e.g., parents, sibling, spouse) burden and associated factors were discussed in ten articles [20, 24, 53, 64, $86,103,111,121,139,172]$, although only four provided prevalence rates $[64,86,103,111]$ for aspects of psychosocial burden. Overall QoL among caregivers was impaired, with depression, anxiety, emotional distress, and impaired social life commonly mentioned. There were no notable consistencies across studies regarding factors affecting caregiver burden. Two studies reported significant parental depression vs controls [24, 121], and one study showed that caregiver depression and anxiety significantly reduced QoL among patients with vitiligo [111].

\section{Discussion}

In the past decade, interest in and publication of the overall and psychosocial QoL of patients with vitiligo have increased tremendously, highlighting the QoL burden in vitiligo. Several recent studies have reported meta-analyses of depression and/or anxiety in patients with vitiligo [10-12], with less focus on other psychosocial comorbidities experienced by patients with vitiligo. We sought to comprehensively review the prevalence of any psychosocial comorbidity reported by patients in peer-reviewed scientific articles.
Studies in this systematic review reported wide ranges (likely owing to differing assessment tools and geographically heterogeneous populations) for the majority of psychosocial comorbidities. Psychosocial comorbidities reported in $>50 \%$ of patients in any study were depression, major depressive disorder, anxiety, social phobia, feelings of stigmatization, adjustment disorders, sleep disturbances, avoidance and restriction behavior, self-consciousness, emotional impairment, relationship difficulties, and cognitive impairment. Psychosocial comorbidities reported in $>25 \%$ of patients included coexistent depression and anxiety, sexual dysfunction, alexithymia, anger, suicidality (unspecified suicidality and suicidal ideation), and dysthymic disorders. The breadth and severity of these comorbidities and the resulting effect on QoL in patients with vitiligo extend beyond what has previously been dismissed as a cosmetic disease.

Factors that were most commonly associated with significantly higher psychosocial burden included female sex, visible or genital lesions, age $<30$ years (particularly adolescents), and extensive body area involvement, among others. The implementation of facial vitiligo as a primary outcome measure in recent clinical studies [178-181] is supported by the gravity of the association between facial lesions and a higher psychosocial burden reported here. Some of the factors significantly associated with a higher psychosocial burden have been associated with a greater willingness to pay, although the association between willingness to pay and lesion location was not assessed [4].

In many cases, studies reported findings using broad QoL instruments that are not specific to vitiligo. Generic QoL instruments may not reflect the true burden of vitiligo, in part because of instrument design. For example, the DLQI includes an item for physical symptoms (i.e., itch, soreness, pain, or stinging) [182], which tend to be more pronounced in patients with atopic dermatitis or psoriasis, possibly leading to an underestimation of burden in vitiligo vs other dermatologic diseases [183, 184]. In addition, the heterogeneity of studies included in this review may further complicate direct comparisons of general QoL in vitiligo with other dermatologic diseases. Interestingly, a recent study in South Korea showed that willingness to pay was highest in vitiligo compared with other dermatologic diseases including atopic dermatitis and psoriasis, despite lower median DLQI scores in patients with vitiligo [27]. The application of widely used generic QoL instruments in vitiligo may therefore be better suited for comparison across demographic or clinical characteristics. In our analysis, there were regional trends in DLQI scores, with a lower QoL burden among European and North American populations and a higher QoL burden noted among Middle Eastern and Asian populations, consistent with 
findings from another review [185]. Future studies should assess QoL instruments for cultural sensitivity/influence. Psychosocial morbidity should also be examined to further elucidate the effect of culture on vitiligo burden, which is an important consideration for dermatologists caring for diverse patients. During the past decade, the VitiQoL was developed specifically for measuring QoL in patients with vitiligo [184], although it does not differentiate between skin types (i.e., fair and dark skin), and its use has not been widespread. Our search criteria identified only eight studies that reported aspects of psychosocial comorbidity using the VitiQoL instrument [13, 16, 29, 32, 67, 80, 88, 115], two of which were clinical trials published in the past year [16, 29]. Further research using vitiligo-specific QoL instruments is warranted.

It is well recognized that more effective treatment strategies are needed for vitiligo. The results of this systematic review raise the urgency for strategies (including better treatments, counseling, and cognitive behavioral therapy) to improve the overall QoL and psychosocial health of patients. Although not directly assessed in this systematic review, the significant association of longer disease duration with poorer psychosocial and general QoL supports the possibility that delayed interventions could exacerbate disease burden. Furthermore, this review highlights the unmet need for a widely used vitiligo-specific instrument to assess psychosocial burden and reinforce that vitiligo is not a purely cosmetic disease. A cross-culturally validated, vitiligo-specific instrument, the 12-item short-form of the Vitiligo Impact Patients scale (VIPs-12), was recently developed to address QoL burden, including psychological effects on daily life and items specifically related to skin type; however, the instrument still awaits testing in prospective studies for responsiveness [186]. In addition to psychotherapy and/or counseling for patients, general education about vitiligo in the unaffected population may help lessen the stigma associated with vitiligo and assist in improving the psychosocial well-being of patients and their caregivers. In surveys among people without vitiligo, participants with sufficient knowledge of the disease were more likely to display positive attitudes toward patients with vitiligo compared with people who had insufficient disease knowledge $[31,78,91]$. The most commonly reported coping strategy among patients in our analysis was concealment of lesions through clothing choices, camouflage, and altered body movements. It may follow that if patients were made to feel more comfortable in their own skin and around others, and if nonpatients were educated to be more accepting, the psychosocial burden among patients with vitiligo and their caregivers could be lessened.

Limitations to this study include the heterogeneity of studies (together with differences in methods used to quantify the presence of psychosocial comorbidities), the paucity of details available in some publications, and restriction to English language in the search criteria. Additionally, inclusion of only peer-reviewed publications may be associated with publication bias [187].

\section{Conclusions}

Vitiligo has a significant and broad effect on psychosocial well-being, an aspect of QoL that may not be accurately or fully captured by currently available QoL instruments. The extent of the psychosocial comorbidities summarized in this systematic review indicates that multidisciplinary approaches to treatment strategies (including medical and psychological treatment) and education about vitiligo are needed to address the burden of this disease.

Supplementary Information The online version contains supplementary material available at https://doi.org/10.1007/s40257-021-00631-6.

Acknowledgements Writing assistance was provided by Wendy van der Spuy, PhD, and Ken Wannemacher, PhD, of ICON (North Wales, PA, USA) and was funded by Incyte Corporation (Wilmington, DE, USA).

\section{Declarations}

Funding The study was funded by Incyte Corporation, Wilmington, DE, USA.

Conflicts of Interest/Competing Interests $\mathrm{KE}$ is a consultant for AbbVie, Incyte Corporation, La Roche-Posay, Pfizer, Pierre Fabre, Sanofi, and Viela Bio. VE has nothing to disclose. HJ and FIK were employees and shareholders of Incyte Corporation when the study was conducted. KB and DS are employees and shareholders of Incyte Corporation. AGP has served as an investigator for Aclaris Therapeutics, Immune Tolerance Network, Incyte Corporation, and Pfizer; a consultant for Arcutis, Avita, Chromaderm, Immune Tolerance Network, Incyte Corporation, Pfizer, Viela Bio, and Villaris; and a board member who also holds stock options for Clarify Medical and Tara Medical.

Ethics Approval Because data were collected from published articles, no ethical approval was required for the study.

Consent to Participate Not applicable.

Consent for Publication Not applicable.

Availability of Data and Material All data were collected from published articles available in the public domain.

Code availability Not applicable.

Authors' Contributions KE, VE, HJ, KB, FIK, DS, and AGP contributed to the study design, including formulation of the search strategy, had access to extracted data, and contributed to data interpretation. KE, VE, HJ, KB, FIK, DS, and AGP were involved in drafting the manuscript. KE, VE, HJ, KB, FIK, DS, and AGP approved the final version for submission and agree to be accountable for all aspects of the work. 
Prior Presentations Data in this article were previously presented, in part, at the European Academy of Dermatology and Venereology 2020 Virtual Congress (29-31 October, 2020) and the Vitiligo International Symposium 2020 Virtual Conference (5-6 December, 2020).

Open Access This article is licensed under a Creative Commons Attribution-NonCommercial 4.0 International License, which permits any non-commercial use, sharing, adaptation, distribution and reproduction in any medium or format, as long as you give appropriate credit to the original author(s) and the source, provide a link to the Creative Commons licence, and indicate if changes were made. The images or other third party material in this article are included in the article's Creative Commons licence, unless indicated otherwise in a credit line to the material. If material is not included in the article's Creative Commons licence and your intended use is not permitted by statutory regulation or exceeds the permitted use, you will need to obtain permission directly from the copyright holder. To view a copy of this licence, visit http://creativecommons.org/licenses/by-nc/4.0/.

\section{References}

1. Taieb A, Picardo M. The definition and assessment of vitiligo: a consensus report of the Vitiligo European Task Force. Pigment Cell Res. 2007;20(1):27-35.

2. Ezzedine K, Sheth V, Rodrigues M, Eleftheriadou V, Harris JE, Hamzavi IH, et al. Vitiligo is not a cosmetic disease. J Am Acad Dermatol. 2015;73(5):883-5.

3. Ezzedine K, Eleftheriadou V, Whitton M, van Geel N. Vitiligo. Lancet. 2015;386(9988):74-84.

4. Radtke MA, Schafer I, Gajur A, Langenbruch A, Augustin M. Willingness-to-pay and quality of life in patients with vitiligo. Br J Dermatol. 2009;161(1):134-9.

5. Talsania N, Lamb B, Bewley A. Vitiligo is more than skin deep: a survey of members of the Vitiligo Society. Clin Exp Dermatol. 2010;35(7):736-9.

6. Kruger C, Schallreuter KU. A review of the worldwide prevalence of vitiligo in children/adolescents and adults. Int J Dermatol. 2012;51(10):1206-12.

7. Morrison B, Burden-Teh E, Batchelor JM, Mead E, Grindlay D, Ratib S. Quality of life in people with vitiligo: a systematic review and meta-analysis. Br J Dermatol. 2017;177(6):e338-9.

8. Cupertino F, Niemeyer-Corbellini JP, Ramos ESM. Psychosomatic aspects of vitiligo. Clin Dermatol. 2017;35(3):292-7.

9. Yang Y, Zapata L, Rodgers C, Hernandez K, Iyer M, Jia G, et al. Quality of life in patients with vitiligo using the Short Form-36. Br J Dermatol. 2017;177(6):1764-6.

10. Lai YC, Yew YW, Kennedy C, Schwartz RA. Vitiligo and depression: a systematic review and meta-analysis of observational studies. Br J Dermatol. 2017;177(3):708-18.

11. Osinubi O, Grainge MJ, Hong L, Ahmed A, Batchelor JM, Grindlay D, et al. The prevalence of psychological comorbidity in people with vitiligo: a systematic review and meta-analysis. Br J Dermatol. 2018;178(4):863-78.

12. Wang G, Qiu D, Yang H, Liu W. The prevalence and odds of depression in patients with vitiligo: a meta-analysis. J Eur Acad Dermatol Venereol. 2018;32(8):1343-51.

13. Anaba EL, Oaku RI. Prospective cross-sectional study of quality of life of vitiligo patients using a vitiligo specific quality of life instrument. West Afr J Med. 2020;37(7):745-9.

14. Temel A, Bozkurt S, Senol Y, Alpsoy E. Internalized stigma in patients with acne vulgaris, vitiligo, and alopecia areata. Turk J Dermatol. 2019;13(3):109-16.
15. Henning SW, Jaishankar D, Barse LW, Dellacecca ER, Lancki $\mathrm{N}$, Webb K, et al. The relationship between stress and vitiligo: evaluating perceived stress and electronic medical record data. PLoS ONE. 2020;15(1):e0227909.

16. Liu B, Sun Y, Song J, Wu Z. Home vs hospital narrowband UVB treatment by a hand-held unit for new-onset vitiligo: a pilot randomized controlled study. Photodermatol Photoimmunol Photomed. 2020;36(1):14-20.

17. Alharbi MA. Identifying patients at higher risk of depression among patients with vitiligo at outpatient setting. Mater Sociomed. 2020;32(2):108-11.

18. Udaya Kiran K, Potharaju AR, Vellala M, Narasimha RP. Cosmetic camouflage of visible skin lesions enhances life quality indices in leprosy as in vitiligo patients: an effective stigma reduction strategy. Lepr Rev. 2020;91(4):343-52.

19. Ibrahim S, El Mofty M, Mostafa W, Esmat S, Samir N, ElSamanoudy S, et al. Monobenzyl ether of hydroquinone 20 and $40 \%$ cream in depigmentation of patients with vitiligo: a randomized controlled trial. J Egypt Womens Dermatol Soc. 2020;17(3):130-7.

20. Andrade G, Rangu S, Provini L, Putterman E, Gauthier A, Castelo-Soccio L. Childhood vitiligo impacts emotional health of parents: a prospective, cross-sectional study of quality of life for primary caregivers. J Patient Rep Outcomes. 2020;4(1):20.

21. Narayan VS, Uitentuis SE, Luiten RM, Bekkenk MW, Wolkerstorfer A. Patients' perspective on current treatments and demand for novel treatments in vitiligo. J Eur Acad Dermatol Venereol. 2021;35(3):744-8.

22. Dabas G, Vinay K, Parsad D, Kumar A, Kumaran MS. Psychological disturbances in patients with pigmentary disorders: a cross-sectional study. J Eur Acad Dermatol Venereol. 2020;34(2):392-9.

23. Chen CY, Wang WM, Chung $\mathrm{CH}$, Tsao $\mathrm{CH}$, Chien WC, Hung CT. Increased risk of psychiatric disorders in adult patients with vitiligo: a nationwide, population-based cohort study in Taiwan. J Dermatol. 2020;47(5):470-5.

24. Savas Erdogan S, Falay Gur T, Dogan B. Anxiety and depression in pediatric patients with vitiligo and alopecia areata and their parents: a cross-sectional controlled study. J Cosmet Dermatol. 2021;20(7):2232-9.

25. Bassiouny D, Hegazy R, Esmat S, Gawdat HI, Ahmed Ezzat M, Tawfik HA, et al. Cosmetic camouflage as an adjuvant to vitiligo therapies: effect on quality of life. J Cosmet Dermatol. 2021;20(1):159-65.

26. Tan A, Schuster LM, Robbins MA, Holavanahalli R, Pandya AG. Evaluation of the psychosocial impact of a Social Interaction Skills Training (SIST) workshop for patients with vitiligo: a pilot study. J Am Acad Dermatol. 2020;83(2):645-7.

27. Bae JM, Kim JE, Lee RW, Ju HJ, Han JH, Lee JH, et al. Beyond the quality of life: a call for patients' own willingness to pay in chronic skin disease to assess psychosocial burden: a multicenter, cross-sectional prospective survey. J Am Acad Dermatol. 2020. https://doi.org/10.1016/j.jaad.2020.09.088.

28. Sarma N, Chakraborty S, Poojary S, Shashi Kumar BM, Gupta LK, Budamakuntla L, et al. A nationwide, multicentric casecontrol study on vitiligo (MEDEC-V) to elicit the magnitude and correlates. Indian J Dermatol. 2020;65(6):473-82.

29. Batchelor JM, Thomas KS, Akram P, Azad J, Bewley A, Chalmers JR, et al. Home-based narrowband UVB, topical corticosteroid or combination for children and adults with vitiligo: HI-Light Vitiligo three-arm RCT. Health Technol Assess. 2020;24(64):1-128.

30. Hamidizadeh N, Ranjbar S, Ghanizadeh A, Parvizi MM, Jafari $\mathrm{P}$, Handjani F. Evaluating prevalence of depression, anxiety and hopelessness in patients with vitiligo on an Iranian population. Health Qual Life Outcomes. 2020;18(1):20. 
31. Tsadik AG, Teklemedhin MZ, Mehari Atey T, Gidey MT, Desta DM. Public knowledge and attitudes towards vitiligo: a survey in Mekelle City, Northern Ethiopia. Dermatol Res Pract. 2020;2020:3495165.

32. Pun J, Randhawa A, Kumar A, Williams V. The impact of vitiligo on quality of life and psychosocial well-being in a Nepalese population. Dermatol Clin. 2021;39(1):117-27.

33. Yucel D, Sener S, Turkmen D, Altunisik N, Sarac G, Cumurcu HB. Evaluation of the Dermatological Life Quality Index, sexual dysfunction and other psychiatric diseases in patients diagnosed with vitiligo with and without genital involvement. Clin Exp Dermatol. 2021;46(4):669-74.

34. Ucuz I, Altunisik N, Sener S, Turkmen D, Kavuran NA, Marsak $\mathrm{M}$, et al. Quality of life, emotion dysregulation, attention deficit and psychiatric comorbidity in children and adolescents with vitiligo. Clin Exp Dermatol. 2021;46(3):510-5.

35. Silpa-Archa N, Pruksaeakanan C, Angkoolpakdeekul N, Chaiyabutr C, Kulthanan K, Ratta-Apha W, et al. Relationship between depression and quality of life among vitiligo patients: a self-assessment questionnaire-based study. Clin Cosmet Investig Dermatol. 2020;13:511-20.

36. Gupta V, Taneja N, Sati HC, Sreenivas V, Ramam M. Evaluation of "not relevant" responses on the Dermatology Life Quality Index (DLQI) and the DLQI-R scoring modification among Indian patients with vitiligo. Br J Dermatol. 2021;184(1):168-9.

37. Lacerda KAP, Silva LA, Mendonca GS, Guimarães RA, Guilo LA. Association between quality of life and perceived stress in patients with vitiligo: case control study. Biosci J. 2020;36(3):1032-42.

38. Amatya B, Pokhrel DB. Assessment and comparison of quality of life in patients with melasma and vitiligo. Kathmandu Univ Med J (KUMJ). 2019;17(66):114-8.

39. Arýcan Ö, Koç K, Ersoy L. Clinical characteristics in 113 Turkish vitiligo patients. Acta Dermatovenerol Alp Pannonica Adriat. 2008;17(3):129-32.

40. Agarwal G. Vitiligo: an under-estimated problem. Fam Pract. 1998;15(Suppl. 1):S19-23.

41. Ahmad D. A community based study on finding the quality of life of the patients with vitiligo. J Appl Pharm Sci. 2017;7(5):90-3.

42. Ahmed A, Steed L, Burden-Teh E, Shah R, Sanyal S, Tour S, et al. Identifying key components for a psychological intervention for people with vitiligo: a quantitative and qualitative study in the United Kingdom using web-based questionnaires of people with vitiligo and healthcare professionals. J Eur Acad Dermatol Venereol. 2018;32(12):2275-83.

43. Ahmed I, Ahmed S, Nasreen S. Frequency and pattern of psychiatric disorders in patients with vitiligo. J Ayub Med Coll Abbottabad. 2007;19(3):19-21.

44. Ajose FO, Parker RA, Merrall EL, Adewuya AO, Zachariah MP. Quantification and comparison of psychiatric distress in African patients with albinism and vitiligo: a 5-year prospective study. J Eur Acad Dermatol Venereol. 2014;28(7):925-32.

45. Al Robaee AA. Assessment of quality of life in Saudi patients with vitiligo in a medical school in Qassim province, Saudi Arabia. Saudi Med J. 2007;28(9):1414-7.

46. AlGhamdi KM. Beliefs and perceptions of Arab vitiligo patients regarding their condition. Int J Dermatol. 2010;49(10):1141-5.

47. AlGhamdi KM, Moussa NA, Mandil A, Alkofidi M, Madani A, Aldaham N, et al. Public perceptions and attitudes toward vitiligo. J Cutan Med Surg. 2012;16(5):334-40.

48. Al-Harbi M. Prevalence of depression in vitiligo patients. Skinmed. 2013;11(6):327-30.

49. Al-Mubarak L, Al-Mohanna H, Al-Issa A, Jabak M, Mulekar SV. Quality of life in Saudi vitiligo patients. J Cutan Aesthet Surg. 2011;4(1):33-7.
50. AlShahwan MA. The prevalence of anxiety and depression in Arab dermatology patients. J Cutan Med Surg. 2015;19(3):297-303.

51. Al-Shobaili HA. Correlation of clinical efficacy and psychosocial impact on vitiligo patients by excimer laser treatment. Ann Saudi Med. 2014;34(2):115-21.

52. Al-Shobaili HA. Treatment of vitiligo patients by excimer laser improves patients' quality of life. J Cutan Med Surg. 2015;19(1):50-6.

53. Amer AA, McHepange UO, Gao XH, Hong Y, Qi R, Wu Y, et al. Hidden victims of childhood vitiligo: impact on parents' mental health and quality of life. Acta Derm Venereol. 2015;95(3):322-5.

54. Asati DP, Gupta CM, Tiwari S, Kumar S, Jamra V. A hospitalbased study on knowledge and attitude related to vitiligo among adults visiting a tertiary health facility of central India. J Nat Sci Biol Med. 2016;7(1):27-32.

55. Aşkın A, Özkan A, Bıyıklı SE, Tosun A. Could there be a possible link between vitiligo and fibromyalgia syndrome? Turk J Dermatol. 2018;12(2):100-6.

56. Bae JM, Lee SC, Kim TH, Yeom SD, Shin JH, Lee WJ, et al. Factors affecting quality of life in patients with vitiligo: a nationwide study. Br J Dermatol. 2018;178(1):238-44.

57. Balaban ÖD, Atagün Mİ, Özgüven HD, Özsan HH. Psychiatric morbidity in patients with vitiligo. Dusunen Adam. 2011;24(4):306-13.

58. Balieva F, Kupfer J, Lien L, Gieler U, Finlay AY, TomasAragones L, et al. The burden of common skin diseases assessed with the EQ5D: a European multicentre study in 13 countries. Br J Dermatol. 2017;176(5):1170-8.

59. Barisic-Drusko V, Rucevic I. Trigger factors in childhood psoriasis and vitiligo. Coll Antropol. 2004;28(1):277-85.

60. Benros ME, Waltoft BL, Nordentoft M, Ostergaard SD, Eaton WW, Krogh J, et al. Autoimmune diseases and severe infections as risk factors for mood disorders: a nationwide study. JAMA Psychiat. 2013;70(8):812-20.

61. Bhatia B, Kechichian E, Eleftheriadou V, Castelneau JP, Seite $\mathrm{S}$, Meurant JM, et al. Habits and risk perception associated with sun exposure in vitiligo patients according to their participation in a patients' organization. J Eur Acad Dermatol Venereol. 2019;33(3):e100-3.

62. Bidaki R, Majidi N, Moghadam Ahmadi A, Bakhshi H, Sadr Mohammadi R, Mostafavi SA, et al. Vitiligo and social acceptance. Clin Cosmet Investig Dermatol. 2018;11:383-6.

63. Bilgic O, Bilgic A, Akis HK, Eskioglu F, Kilic EZ. Depression, anxiety and health-related quality of life in children and adolescents with vitiligo. Clin Exp Dermatol. 2011;36(4):360-5.

64. Bin Saif GA, Al-Balbeesi AO, Binshabaib R, Alsaad D, Kwatra SG, Alzolibani AA, et al. Quality of life in family members of vitiligo patients: a questionnaire study in Saudi Arabia. Am J Clin Dermatol. 2013;14(6):489-95.

65. Bonotis K, Pantelis K, Karaoulanis S, Katsimaglis C, Papaliaga $\mathrm{M}$, Zafiriou $\mathrm{E}$, et al. Investigation of factors associated with health-related quality of life and psychological distress in vitiligo. J Dtsch Dermatol Ges. 2016;14(1):45-9.

66. Borimnejad L, Parsa Yekta Z, Nikbakht-Nasrabadi A, Firooz A. Quality of life with vitiligo: comparison of male and female Muslim patients in Iran. Gend Med. 2006;3(2):124-30.

67. Catucci Boza J, Giongo N, Machado P, Horn R, Fabbrin A, Cestari T. Quality of life impairment in children and adults with vitiligo: a cross-sectional study based on dermatology-specific and disease-specific quality of life instruments. Dermatology. 2016;232(5):619-25.

68. Chan MF, Chua TL, Goh BK, Aw CW, Thng TG, Lee SM. Investigating factors associated with depression of vitiligo patients in Singapore. J Clin Nurs. 2012;21(11-12):1614-21. 
69. Chan MF, Thng TG, Aw CW, Goh BK, Lee SM, Chua TL. Investigating factors associated with quality of life of vitiligo patients in Singapore. Int J Nurs Pract. 2013;19(Suppl. 3):3-10.

70. Chen D, Tuan H, Zhou EY, Liu D, Zhao Y. Quality of life of adult vitiligo patients using camouflage: a survey in a Chinese vitiligo community. PLoS ONE. 2019;14(1):e0210581.

71. Choi S, Kim DY, Whang SH, Lee JH, Hann SK, Shin YJ. Quality of life and psychological adaptation of Korean adolescents with vitiligo. J Eur Acad Dermatol Venereol. 2010;24(5):524-9.

72. Dehghani F, Dehghani F, Kafaie P, Taghizadeh MR. Alexithymia in different dermatologic patients. Asian J Psychiatr. 2017;25:42-5.

73. Dertlioglu SB, Cicek D, Balci DD, Halisdemir N. Dermatology Life Quality Index scores in children with vitiligo: comparison with atopic dermatitis and healthy control subjects. Int J Dermatol. 2013;52(1):96-101.

74. Dołruk Kaçar S, Özułuz P, Bą̧ciołlu E, Coşkun KŞ, Polat S, Karaca S.. Is a poor Dermatology Life Quality Index score a sign of stigmatization in patients with vitiligo? Turk Clin Dermatol. 2014;24(2):45-50.

75. Erfan G, Albayrak Y, Yanik ME, Oksuz O, Tasolar K, Topcu B, et al. Distinct temperament and character profiles in first onset vitiligo but not in alopecia areata. J Dermatol. 2014;41(8):709-15.

76. Essa N, Awad S, Nashaat M. Validation of an Egyptian Arabic version of Skindex-16 and quality of life measurement in Egyptian patients with skin disease. Int J Behav Med. 2018;25(2):243-51.

77. Ezzedine K, Grimes PE, Meurant JM, Seneschal J, LeauteLabreze C, Ballanger F, et al. Living with vitiligo: results from a national survey indicate differences between skin phototypes. Br J Dermatol. 2015;173(2):607-9.

78. Fatani MI, Aldhahri RM, Al Otaibi HO, Kalo BB, Khalifa MA. Acknowledging popular misconceptions about vitiligo in western Saudi Arabia. J Dermatol Dermatol Surg. 2016;20(1):27-31.

79. Firooz A, Bouzari N, Fallah N, Ghazisaidi B, Firoozabadi MR, Dowlati Y. What patients with vitiligo believe about their condition. Int J Dermatol. 2004;43(11):811-4.

80. Florez-Pollack S, Jia G, Zapata L Jr, Rodgers C, Hernandez $\mathrm{K}$, Hynan LS, et al. Association of quality of life and location of lesions in patients with vitiligo. JAMA Dermatol. 2017;153(3):341-2.

81. Ganguly S, Saha S, Koley M, Mondal R. Homeopathic treatment of vitiligo: an open observational pilot study. Int J High Dilution Res. 2013;12(45):168-77.

82. Ghaderi R, Saadatjoo A. Evaluating of life quality in Iranian patients with vitiligo using generic and special questionnaires. Shiraz E Med J. 2014;15(3):e22359.

83. Ghajarzadeh M, Ghiasi M, Kheirkhah S. Associations between skin diseases and quality of life: a comparison of psoriasis, vitiligo, and alopecia areata. Acta Med Iran. 2012;50(7):511-5.

84. Güler Ö, Karaca Ş, Aşik AH, Yalçin C. Psychosocial symptoms in patients with psoriasis, vitiligo, and neurodermatitis. Neurol Psychiatry Brain Res. 2009;16(3-4):139-44.

85. Gurpinar A, Dogan Gunaydin S, Kilic C, Karaduman A. Association of serum cortisol and dehydroepiandrosterone sulfate (DHEAS) levels with psychological stress in patients with vitiligo. Turk J Med Sci. 2019;49(3):832-7.

86. Handjani F, Kalafi A. Impact of dermatological diseases on family members of the patients using Family Dermatology Life Quality Index: a preliminary study in Iran. Iran J Dermatol. 2013;16(66):128-31.

87. Hassanin AM, Ismail NN, El Guindi A, Sowailam HA. The emotional burden of chronic skin disease dominates physical factors among women, adversely affecting quality of life and sexual function. J Psychosom Res. 2018;115:53-7.
88. Hedayat K, Karbakhsh M, Ghiasi M, Goodarzi A, Fakour Y, Akbari Z, et al. Quality of life in patients with vitiligo: a crosssectional study based on Vitiligo Quality of Life index (VitiQoL). Health Qual Life Outcomes. 2016;14:86.

89. Ingordo $\mathrm{V}$, Cazzaniga S, Gentile C, Iannazzone SS, Cusano F, Naldi L. Dermatology Life Quality Index score in vitiligo patients: a pilot study among young Italian males. G Ital Dermatol Venereol. 2012;147(1):83-90.

90. Ingordo V, Cazzaniga S, Medri M, Raone B, Digiuseppe MD, Musumeci ML, et al. To what extent is quality of life impaired in vitiligo? A multicenter study on Italian patients using the Dermatology Life Quality Index. Dermatology. 2014;229(3):240-7.

91. Juntongjin P, Rachawong C, Nuallaong W. Knowledge and attitudes towards vitiligo in the general population: a survey based on the simulation video of a real situation. Dermatol Sin. 2018;36(2):75-8.

92. Karelson M, Silm H, Kingo K. Quality of life and emotional state in vitiligo in an Estonian sample: comparison with psoriasis and healthy controls. Acta Derm Venereol. 2013;93(4):446-50.

93. Kent G. Correlates of perceived stigma in vitiligo. Psychol Health. 1999;14(2):241-51.

94. Kent G. Understanding the experiences of people with disfigurements: an integration of four models of social and psychological functioning. Psychol Health Med. 2000;5(2):117-29.

95. Kent G, Al'Abadie M. Psychologic effects of vitiligo: a critical incident analysis. J Am Acad Dermatol. 1996;35(6):895-8.

96. Kent G, Al-Abadie M. Factors affecting responses on Dermatology Life Quality Index items among vitiligo sufferers. Clin Exp Dermatol. 1996;21(5):330-3.

97. Kim DY, Lee J, Oh SH, Hann SK, Shin YJ. Impact of genital involvement on the sexual lives of vitiligo patients. J Dermatol. 2013;40(12):1065-7.

98. Kim DY, Lee JW, Whang SH, Park YK, Hann SK, Shin YJ. Quality of life for Korean patients with vitiligo: Skindex-29 and its correlation with clinical profiles. J Dermatol. 2009;36(6):317-22.

99. Kostopoulou P, Jouary T, Quintard B, Ezzedine K, Marques S, Boutchnei S, et al. Objective vs. subjective factors in the psychological impact of vitiligo: the experience from a French referral centre. Br J Dermatol. 2009;161(1):128-33.

100. Kota RS, Vora RV, Varma JR, Kota SK, Patel TM, Ganjiwale J. Study on assessment of quality of life and depression in patients of vitiligo. Indian Dermatol Online J. 2019;10(2):153-7.

101. Kruger C, Panske A, Schallreuter KU. Disease-related behavioral patterns and experiences affect quality of life in children and adolescents with vitiligo. Int J Dermatol. 2014;53(1):43-50.

102. Kruger C, Schallreuter KU. Stigmatisation, avoidance behaviour and difficulties in coping are common among adult patients with vitiligo. Acta Derm Venereol. 2015;95(5):553-8.

103. Krüger C, Schallreuter KU. Increased levels of anxious-depressive mood in parents of children with vitiligo. Eur J Pediatr Dermatol. 2018;28(2):70-8.

104. Kruger C, Smythe JW, Spencer JD, Hasse S, Panske A, Chiuchiarelli G, et al. Significant immediate and long-term improvement in quality of life and disease coping in patients with vitiligo after group climatotherapy at the Dead Sea. Acta Derm Venereol. 2011;91(2):152-9.

105. Linthorst Homan MW, de Korte J, Grootenhuis MA, Bos JD, Sprangers MA, van der Veen JP. Impact of childhood vitiligo on adult life. Br J Dermatol. 2008;159(4):915-20.

106. Linthorst Homan MW, Sprangers MA, de Korte J, Bos JD, van der Veen JP. Characteristics of patients with universal vitiligo and health-related quality of life. Arch Dermatol. 2008;144(8):1062-4.

107. Linthorst Homan MW, Spuls PI, de Korte J, Bos JD, Sprangers MA, van der Veen JP. The burden of vitiligo: patient 
characteristics associated with quality of life. J Am Acad Dermatol. 2009;61(3):411-20.

108. Maghami Z, Sepaskhah M, Sarani EM, Bagheri Z, Maghami M. Alexithymia in vitiligo patients: a case-control study. Iran J Dermatol. 2018;21(4):138-41.

109. Manolache L, Benea V. Stress in patients with alopecia areata and vitiligo. J Eur Acad Dermatol Venereol. 2007;21(7):921-8.

110. Manzoni AP, Pereira RL, Townsend RZ, Weber MB, Nagatomi AR, Cestari TF. Assessment of the quality of life of pediatric patients with the major chronic childhood skin diseases. An Bras Dermatol. 2012;87(3):361-8.

111. Manzoni AP, Weber MB, Nagatomi AR, Pereira RL, Townsend RZ, Cestari TF. Assessing depression and anxiety in the caregivers of pediatric patients with chronic skin disorders. An Bras Dermatol. 2013;88(6):894-9.

112. Mashayekhi V, Javidi Z, Kiafar B, Manteghi AA, Saadatian V, Esmaeili HA, et al. Quality of life in patients with vitiligo: a descriptive study on 83 patients attending a PUVA therapy unit in Imam Reza Hospital, Mashad. Indian J Dermatol Venereol Leprol. 2010;76(5):592.

113. Mattoo SK, Handa S, Kaur I, Gupta N, Malhotra R. Psychiatric morbidity in vitiligo and psoriasis: a comparative study from India. J Dermatol. 2001;28(8):424-32.

114. Mattoo SK, Handa S, Kaur I, Gupta N, Malhotra R. Psychiatric morbidity in vitiligo: prevalence and correlates in India. J Eur Acad Dermatol Venereol. 2002;16(6):573-8.

115. Morales-Sanchez MA, Vargas-Salinas M, Peralta-Pedrero ML, Olguin-Garcia MG, Jurado-Santa Cruz F. Impact of vitiligo on quality of life. Actas Dermosifiliogr. 2017;108(7):637-42.

116. Moretti S, Arunachalam M, Colucci R, Pallanti S, Kline JA, Berti $\mathrm{S}$, et al. Autoimmune markers in vitiligo patients appear correlated with obsession and phobia. J Eur Acad Dermatol Venereol. 2012;26(7):861-7.

117. Mouzas O, Angelopoulos N, Papaliagka M, Tsogas P. Increased frequency of self-reported parasomnias in patients suffering from vitiligo. Eur J Dermatol. 2008;18(2):165-8.

118. Nijsten T, Sampogna F, Abeni D. Categorization of Skindex-29 scores using mixture analysis. Dermatology. 2009;218(2):151-4.

119. Noh S, Kim M, Park CO, Hann SK, Oh SH. Comparison of the psychological impacts of asymptomatic and symptomatic cutaneous diseases: vitiligo and atopic dermatitis. Ann Dermatol. 2013;25(4):454-61.

120. Noor SM, Khurshid K, Mahmood T, Haroon TS. Quality of life in vitiligo patients. J Pakistan Assoc Dermatol. 2004;14(2):55-8.

121. Önen Ö, Kundak S, Özek Erkuran H, Kutlu A, Çakaloz B. Quality of life, depression, and anxiety in Turkish children with vitiligo and their parents. Psychiat Clin Psych. 2018;2018:1-10.

122. Ongenae K, Dierckxsens L, Brochez L, van Geel N, Naeyaert JM. Quality of life and stigmatization profile in a cohort of vitiligo patients and effect of the use of camouflage. Dermatology. 2005;210(4):279-85.

123. Ongenae K, Van Geel N, De Schepper S, Naeyaert JM. Effect of vitiligo on self-reported health-related quality of life. $\mathrm{Br} \mathrm{J}$ Dermatol. 2005;152(6):1165-72.

124. Osman AM, Elkordufani Y, Abdullah MA. The psychological impact of vitiligo in adult Sudanese patients. Afr J Psychiatry (Johannesbg). 2009;12(4):284-6.

125. Papadopoulos L, Bor R, Legg C. Coping with the disfiguring effects of vitiligo: a preliminary investigation into the effects of cognitive-behavioural therapy. Br J Med Psychol. 1999;72(Pt 3):385-96.

126. Papadopoulos L, Bor R, Walker C, Flaxman P, Legg C. Different shades of meaning: illness beliefs among vitiligo sufferers. Psychol Health Med. 2002;7(4):425-33.
127. Papadopoulos L, Walker C, Anthis L. Living with vitiligo: a controlled investigation into the effects of group cognitivebehavioural and person-centred therapies. Dermatol Psychosom. 2004;5(4):172-7.

128. Patel KR, Singam V, Rastogi S, Lee HH, Silverberg NB, Silverberg JI. Association of vitiligo with hospitalization for mental health disorders in US adults. J Eur Acad Dermatol Venereol. 2019;33(1):191-7.

129. Picardi A, Pasquini P, Cattaruzza MS, Gaetano P, Melchi CF, Baliva G, et al. Stressful life events, social support, attachment security and alexithymia in vitiligo: a case-control study. Psychother Psychosom. 2003;72(3):150-8.

130. Pichaimuthu R, Ramaswamy P, Bikash K, Joseph R. A measurement of the stigma among vitiligo and psoriasis patients in India. Indian J Dermatol Venereol Leprol. 2011;77(3):300-6.

131. Porter J, Beuf AH, Lerner A, Nordlund J. Response to cosmetic disfigurement: patients with vitiligo. Cutis. 1987;39(6):493-4.

132. Porter J, Beuf AH, Nordlund JJ, Lerner AB. Psychological reaction to chronic skin disorders: a study of patients with vitiligo. Gen Hosp Psychiatry. 1979;1(1):73-7.

133. Porter JR, Beuf AH. Racial variation in reaction to physical stigma: a study of degree of disturbance by vitiligo among black and white patients. J Health Soc Behav. 1991;32(2):192-204.

134. Porter JR, Beuf AH, Lerner A, Nordlund J. Psychosocial effect of vitiligo: a comparison of vitiligo patients with "normal" control subjects, with psoriasis patients, and with patients with other pigmentary disorders. J Am Acad Dermatol. 1986;15(2 Pt 1):220-4.

135. Porter JR, Beuf AH, Lerner AB, Nordlund JJ. The effect of vitiligo on sexual relationships. J Am Acad Dermatol. 1990;22(2 Pt 1):221-2

136. Premkumar R, Kar B, Rajan P, Richard J. Major precipitating factors for stigma among stigmatized vitiligo and psoriasis patients with brown-black skin shades. Indian J Dermatol Venereol Leprol. 2013;79(5):703-5.

137. Raikhy S, Gautam S, Kanodia S. Pattern and prevalence of psychiatric disorders among patients attending dermatology OPD. Asian J Psychiatr. 2017;29:85-8.

138. Ramakrishna P, Rajni T. Psychiatric morbidity and quality of life in vitiligo patients. Indian J Psychol Med. 2014;36(3):302-3.

139. Saeedeh F, Hossein S, Saman M, Maryam K, Rezvan A, Mahin A, et al. Evaluation of quality of life in parents of the patients with vitiligo by Persian version of the Family Dermatology Life Quality Index (FDLQI) in Kerman. J Pakistan Assoc Dermatol. 2019;29(2):196-202.

140. Salman A, Kurt E, Topcuoglu V, Demircay Z. Social anxiety and quality of life in vitiligo and acne patients with facial involvement: a cross-sectional controlled study. Am J Clin Dermatol. 2016;17(3):305-11.

141. Salzer BA, Schallreuter KU. Investigation of the personality structure in patients with vitiligo and a possible association with impaired catecholamine metabolism. Dermatology. 1995;190(2):109-15.

142. Sampogna F, Raskovic D, Guerra L, Pedicelli C, Tabolli S, Leoni L, et al. Identification of categories at risk for high quality of life impairment in patients with vitiligo. Br J Dermatol. 2008;159(2):351-9.

143. Sangma LN, Nath J, Bhagabati D. Quality of life and psychological morbidity in vitiligo patients: a study in a teaching hospital from north-East India. Indian J Dermatol. 2015;60(2):142-6.

144. Sarhan D, Mohammed GF, Gomaa AH, Eyada MM. Female genital dialogues: female genital self-image, sexual dysfunction, and quality of life in patients with vitiligo with and without genital affection. J Sex Marital Ther. 2016;42(3):267-76.

145. Sarkar S, Sarkar T, Sarkar A, Das S. Vitiligo and psychiatric morbidity: a profile from a vitiligo clinic of a rural-based tertiary care center of Eastern India. Indian J Dermatol. 2018;63(4):281-4. 
146. Sawant NS, Vanjari NA, Khopkar U. Gender differences in depression, coping, stigma, and quality of life in patients of vitiligo. Dermatol Res Pract. 2019;2019:6879412.

147. Schmid-Ott G, Kunsebeck HW, Jecht E, Shimshoni R, Lazaroff I, Schallmayer S, et al. Stigmatization experience, coping and sense of coherence in vitiligo patients. J Eur Acad Dermatol Venereol. 2007;21(4):456-61.

148. Shah R, Hunt J, Webb TL, Thompson AR. Starting to develop self-help for social anxiety associated with vitiligo: using clinical significance to measure the potential effectiveness of enhanced psychological self-help. Br J Dermatol. 2014;171(2):332-7.

149. Shaker OG, Eltahlawi SM, Tawfic SO, Eltawdy AM, Bedair NI. Corticotropin-releasing hormone $(\mathrm{CRH})$ and $\mathrm{CRH}$ receptor 1 gene expression in vitiligo. Clin Exp Dermatol. 2016;41(7):734-40.

150. Sharma N, Koranne RV, Singh RK. Psychiatric morbidity in psoriasis and vitiligo: a comparative study. J Dermatol. 2001;28(8):419-23.

151. Silverberg JI, Silverberg NB. Association between vitiligo extent and distribution and quality-of-life impairment. JAMA Dermatol. 2013;149(2):159-64.

152. Silverberg JI, Silverberg NB. Quality of life impairment in children and adolescents with vitiligo. Pediatr Dermatol. 2014;31(3):309-18.

153. Silverberg JI, Silverberg NB. Vitiligo disease triggers: psychological stressors preceding the onset of disease. Cutis. 2015;95(5):255-62.

154. Sorour F, Abdelmoaty A, Bahary MH, El Birqdar B. Psychiatric disorders associated with some chronic dermatologic diseases among a group of Egyptian dermatology outpatient clinic attendants. J Egypt Womens Dermatol Soc. 2017;14(1):31-6.

155. Sukan M, Maner F. The problems in sexual functions of vitiligo and chronic urticaria patients. J Sex Marital Ther. 2007;33(1):55-64.

156. Tanioka M, Yamamoto Y, Kato M, Miyachi Y. Camouflage for patients with vitiligo vulgaris improved their quality of life. J Cosmet Dermatol. 2010;9(1):72-5.

157. Tjioe M, Otero ME, van de Kerkhof PC, Gerritsen MJ. Quality of life in vitiligo patients after treatment with long-term narrowband ultraviolet B phototherapy. J Eur Acad Dermatol Venereol. 2005;19(1):56-60.

158. Topal IO, Duman H, Goncu OE, Durmuscan M, Gungor S, Ulkumen PK. Knowledge, beliefs, and perceptions of Turkish vitiligo patients regarding their condition. An Bras Dermatol. 2016;91(6):770-5.

159. Tsintsadze N, Beridze L, Tsintsadze N, Krichun Y, Tsivadze N, Tsintsadze M. Psychosomatic aspects in patients with dermatologic diseases. Georgian Med News. 2015;243:70-5.

160. Vallerand IA, Lewinson RT, Parsons LM, Hardin J, Haber RM, Lowerison MW, et al. Vitiligo and major depressive disorder: a bidirectional population-based cohort study. J Am Acad Dermatol. 2019;80(5):1371-9.

161. Wang KY, Wang KH, Zhang ZP. Health-related quality of life and marital quality of vitiligo patients in China. J Eur Acad Dermatol Venereol. 2011;25(4):429-35.

162. Xu ST, Oh EH, Kim JE, Ko JY, Ro YS. Comparative study of quality of life between psoriasis, vitiligo and autoimmune bullous disease. Hong Kong J Dermatol Venereol. 2017;25(2):57-64.

163. Yamamoto Y, Tanioka M, Hayashino Y, Mishina H, Kato M, Fukuhara S, et al. Application of a two-question screening instrument to detect depressive symptoms in patients with vitiligo: a pilot study. J Am Acad Dermatol. 2011;64(5):e69-70.

164. Yanik ME, Erfan G, Albayrak Y, Aydin M, Kulac M, Kuloglu M. Reduced serum brain-derived neurotrophic factor in patients with first onset vitiligo. Neuropsychiatr Dis Treat. 2014;10:2361-7.
165. Zabetian S, Jacobson G, Lim HW, Eide MJ, Huggins RH. Quality of life in a vitiligo support group. J Drugs Dermatol. 2017;16(4):344-50.

166. Zandi S, Farajzadeh S, Saberi N. Effect of vitiligo on self reported quality of life in southern part of Iran. J Pakistan Assoc Dermatol. 2011;21(1):4-9.

167. Papadopoulos L, Bor R, Legg C, Hawk JL. Impact of life events on the onset of vitiligo in adults: preliminary evidence for a psychological dimension in aetiology. Clin Exp Dermatol. 1998;23(6):243-8.

168. Jha A, Mehta M, Khaitan BK, Sharma VK, Ramam M. Cognitive behavior therapy for psychosocial stress in vitiligo. Indian J Dermatol Venereol Leprol. 2016;82(3):308-10.

169. Thompson AR, Clarke SA, Newell RJ, Gawkrodger DJ, Appearance Research Collaboration. Vitiligo linked to stigmatization in British South Asian women: a qualitative study of the experiences of living with vitiligo. Br J Dermatol. 2010;163(3):481-6.

170. Thompson AR, Kent G, Smith JA. Living with vitiligo: dealing with difference. Br J Health Psychol. 2002;7(Pt 2):213-25.

171. Penha MA, Santos PM, Miot HA. Dimensioning the fear of dermatologic diseases. An Bras Dermatol. 2012;87(5):796-9.

172. Pahwa P, Mehta M, Khaitan BK, Sharma VK, Ramam M. The psychosocial impact of vitiligo in Indian patients. Indian J Dermatol Venereol Leprol. 2013;79(5):679-85.

173. Ray A, Pal MK, Ghosh M, Sanyal D. Psychodermatological disorders: an assessment of psychiatric morbidity. Int Med J. 2011;18(4):300-4.

174. Sanclemente G, Burgos C, Nova J, Hernandez F, Gonzalez C, Reyes MI, et al. The impact of skin diseases on quality of life: a multicenter study. Actas Dermosifiliogr. 2017;108(3):244-52.

175. Sampogna F, Picardi A, Chren MM, Melchi CF, Pasquini P, Masini C, et al. Association between poorer quality of life and psychiatric morbidity in patients with different dermatological conditions. Psychosom Med. 2004;66(4):620-4.

176. Hill-Beuf A, Porter JD. Children coping with impaired appearance: social and psychologic influences. Gen Hosp Psychiatry. 1984;6(4):294-301.

177. Kosaraju SK, Reddy KS, Vadlamani N, Sandhya L, Kalasapati L, Maganti S, et al. Psychological morbidity among dermatological patients in a rural setting. Indian J Dermatol. 2015;60(6):635.

178. Rosmarin D, Pandya AG, Lebwohl M, Grimes P, Hamzavi I, Gottlieb $\mathrm{AB}$, et al. Ruxolitinib cream for treatment of vitiligo: a randomised, controlled, phase 2 trial. Lancet. 2020;396(10244):110-20.

179. Kanokrungsee S, Pruettivorawongse D, Rajatanavin N. Clinical outcomes of topical bimatoprost for nonsegmental facial vitiligo: a preliminary study. J Cosmet Dermatol. 2021;20(3):812-8.

180. Ju HJ, Kim SH, Lee JH, Kim GM, Bae JM. Efficacy and safety of tretinoin $0.05 \%$ cream to prevent hyperpigmentation during narrowband UV-B phototherapy in patients with facial vitiligo: a randomized clinical trial. J Dermatolog Treat. 2020;2020:1-4.

181. Seneschal J, Duplaine A, Maillard H, Passeron T, Andreu N, Lassalle R, et al. Efficacy and safety of tacrolimus $0.1 \%$ for the treatment of facial vitiligo: a multicenter randomized, double-blinded, vehicle-controlled study. J Invest Dermatol. 2021;141(7):1728-34.

182. Finlay AY, Khan GK. Dermatology Life Quality Index (DLQI): a simple practical measure for routine clinical use. Clin Exp Dermatol. 1994;19(3):210-6.

183. Gawkrodger DJ, Ormerod AD, Shaw L, Mauri-Sole I, Whitton ME, Watts MJ, et al. Guideline for the diagnosis and management of vitiligo. Br J Dermatol. 2008;159(5):1051-76.

184. Lilly E, Lu PD, Borovicka JH, Victorson D, Kwasny MJ, West DP, et al. Development and validation of a vitiligo-specific quality-of-life instrument (VitiQoL). J Am Acad Dermatol. 2013;69(1):e11-8. 
185. Amer AA, Gao XH. Quality of life in patients with vitiligo: an analysis of the Dermatology Life Quality Index outcome over the past two decades. Int J Dermatol. 2016;55(6):608-14.

186. Ezzedine K, Ahmed M, Tovar-Garza A, Hajj C, Whitton M, Pandya A, et al. Cross-cultural validation of a short-form of the Vitiligo Impact Patient scale (VIPs). J Am Acad Dermatol. 2019;81(5):1107-14.

187. Drucker AM, Fleming P, Chan AW. Research techniques made simple: assessing risk of bias in systematic reviews. J Invest Dermatol. 2016;136(11):e109-14.

\section{Authors and Affiliations}

\section{Khaled Ezzedine ${ }^{1} \cdot$ Viktoria Eleftheriadou $^{2} \cdot$ Heather Jones $^{3} \cdot$ Kristen Bibeau $^{3} \cdot$ Fiona I. Kuo $^{3} \cdot$ Daniel Sturm $^{3}$. Amit G. Pandya ${ }^{4,5}$}

Khaled Ezzedine

khaled.ezzedine@aphp.fr

1 Department of Dermatology, Henri Mondor University Hospital and Université Paris-Est Créteil Val de Marne, 51 Avenue du Maréchal de Lattre de Tassigny, Créteil, 94010 Paris, France

2 Department of Dermatology, Queen Elizabeth Hospital, Birmingham, UK
Incyte Corporation, Wilmington, DE, USA

4 Palo Alto Foundation Medical Group, Mountain View, CA, USA

5 University of Texas Southwestern Medical Center, Dallas, TX, USA 